\title{
SUR LES REPRESENTATIONS UNITAIRES DES GROUPES DE LIE NILPOTENTS. VI
}

\author{
JACQUES DIXMIER
}

Soient $\Gamma$ un groupe localement compact, $\bar{\Gamma}$ l'ensemble des classes de représentations unitaires irréductibles de $\Gamma$. Dans (4), Godement a défini une topologie sur $\bar{\Gamma}$ que nous appellerons "topologie canonique." Cette topologie est facile à étudier lorsque $\Gamma$ est abélien ou compact, mais fort mal connue en général. Dans un article récent (3), Fell a repris l'étude de la topologie canonique de $\bar{\Gamma}$, et réussi à la calculer lorsque $\Gamma$ est le groupe spécial linéaire complexe à $n$ variables. L'espace $\bar{\Gamma}$ est alors "presque" séparé, et, par suite, "presque" localement compact.

Il semble nécessaire d'étudier complètement quelques cas particuliers avant d'entreprendre une théorie générale de $\bar{\Gamma}$. Dans un article antérieur (1), j'ai déterminé l'ensemble $\bar{\Gamma}$ lorsque $\Gamma$ est un groupe de Lie nilpotent simplement connexe de dimension $\leqslant 5$ (il y a essentiellement 8 tels groupes notés $\Gamma_{3}, \Gamma_{4}$, $\Gamma_{5,1}, \Gamma_{5,2}, \ldots, \Gamma_{5,6}$ dans (1); nous conserverons ces notations ici). Nous allons calculer les topologies canoniques de $\bar{\Gamma}_{3}, \bar{\Gamma}_{4}, \bar{\Gamma}_{5,1}, \bar{\Gamma}_{5,2}, \bar{\Gamma}_{5,3}$, et $\bar{\Gamma}_{5,6}$, et obtenir un résultat partiel pour $\bar{\Gamma}_{5,5}$ (le cas de $\Gamma_{5,4}$ conduit à des calculs qui m'ont rebuté). Bien que l'écart entre les topologies trouvées et la séparation soit plus sévère que dans le cas de Fell, les espaces obtenus sont encore très raisonnables. La méthode des éléments "boundedly represented" de Fell ne semble pas utilisable dans notre cas; mais il nous suffira d'appliquer le premier chapitre de (3), en le combinant avec quelques calculs explicites, assez variables d'un cas à l'autre.

Les résultats conduisent à un certain nombre de conjectures. Par exemple:

(1) Soient $\Gamma$ un groupe de Lie nilpotent simplement connexe, $\mathfrak{g}$ son algèbre de Lie, $\mathfrak{U}(\mathfrak{g})$ l'algèbre enveloppante de $\mathfrak{g}, \boldsymbol{Z}(\mathfrak{g})$ le centre de $\mathfrak{U}(\mathfrak{g})$. Tout $a \in Z(\mathfrak{g})$ définit une fonction scalaire $f_{a}$ sur $\bar{\Gamma}$. Alors $f_{a}$ est continue. (Ceci est maintenant démontré; cf. P. Bernat et J. Dixmier, C. R. Acad. Sci. Paris, 1960).

(2) Soit $P$ l'ensemble des points $U$ de $\bar{\Gamma}$ possédant la propriété suivante: pour tout $V \in \bar{\Gamma}, V \neq U$, il existe des voisinages de $U$ et $V$ qui sont disjoints. Alors, $P$ est une partie ouverte partout dense de $\bar{\Gamma}$, et la topologie induite sur $P$ par la topologie canonique de $\bar{\Gamma}$ est la moins fine rendant continues les fonctions $f_{a}$.

D'après (2), les caractères des groupes nilpotents simplement connexes sont des distributions tempérées. Nous les calculons pour $\Gamma_{4}$ et $\Gamma_{5,5}$, ce qui fait apparaître des fonctions de Bessel.

Notations. On désignera par $\mathbf{R}$ le corps des nombres réels, par $\mathbf{C}$ le corps

Reçu le 17 mars, 1959. 
des nombres complexes, par $L_{\mathbf{C}}{ }^{2}\left(\mathbf{R}^{n}\right)$ l'ensemble des fonctions complexes sur $\mathbf{R}^{n}$ de carré intégrable pour la mesure de Lebesgue, par $\mathscr{S}\left(\mathbf{R}^{n}\right)$ l'ensemble des fonctions complexes sur $\mathbf{R}^{n}$ indéfiniment différentiables à décroissance rapide. $\mathrm{Si} \Gamma$ est un groupe de Lie nilpotent simplement connexe, il y a une notion intrinsèque de fonction polynôme sur $\Gamma$, donc $\mathscr{S}(\Gamma)$ a encore un sens. On désignera par $\mathscr{F}$ la transformation de Fourier. Si $F \in \mathscr{S}\left(\mathbf{R}^{4}\right)$, on notera $\hat{F}_{24}$ la fonction

$$
\left(\xi_{1}, \xi_{2}, \xi_{3}, \xi_{4}\right) \rightarrow \iint F\left(\xi_{1}, \eta_{2}, \xi_{3}, \eta_{4}\right) \exp -i\left(\xi_{2} \eta_{2}+\xi_{4} \eta_{4}\right) d \eta_{2} d \eta_{4},
$$

transformée de Fourier de $F$ par rapport aux 2e et 4 e variables; et on définira de manière analogue, pour $F \in \mathscr{S}\left(\mathbf{R}^{n}\right)$, la fonction

$$
\hat{F}_{i_{1} i_{2} \ldots i_{p}} \text { pour } 1 \leqslant i_{1}<i_{2}<\ldots<i_{p} \leqslant n .
$$

Si $\beta_{1}, \beta_{2}, \ldots, \beta_{n} \in \mathbf{R}$, on notera $\mathscr{T}\left(\beta_{1}, \ldots, \beta_{n}\right)$ l'opérateur unitaire dans $L_{\mathbf{C}^{2}}\left(\mathbf{R}^{n}\right)$ défini par

$$
\left(\mathscr{T}\left(\beta_{1}, \ldots, \beta_{n}\right) f\right) \quad\left(\xi_{1}, \ldots, \xi_{n}\right)=f\left(\xi_{1}+\beta_{1}, \ldots, \xi_{n}+\beta_{n}\right) .
$$

Si $h$ est une fonction complexe mesurable essentiellement bornée sur $\mathbf{R}^{n}$, on notera $\mathscr{M}(h)$ (ou $\mathscr{M}\left(h\left(\xi_{1}, \ldots, \xi_{n}\right)\right)$ par abus de notation) l'opérateur continu dans $L_{\mathbf{G}}{ }^{2}\left(\mathbf{R}^{n}\right)$ défini par

$$
(\mathscr{M}(h) f)\left(\xi_{1}, \ldots, \xi_{n}\right)=h\left(\xi_{1}, \ldots, \xi_{n}\right) f\left(\xi_{1}, \ldots, \xi_{n}\right) .
$$

\section{Quelques lemmes.}

Lemme 1. Soient $A$ une $C^{*}$-algèbre, $B$ une partie partout dense de $A$; soient $T_{0}$ un point $d u$ spectre $\hat{A}$ de $A$, et $S \subset \hat{A}$. On suppose que, pour tout $x \in B$, on $a \sup _{T \in S}\|T(x)\| \geqslant\left\|T_{0}(x)\right\|$. Alors $T_{0}$ est canoniquement adhérent $\grave{a} S$.

Démonstration. L'inégalité $\sup _{T \epsilon S}\|T(x)\| \geqslant\left\|T_{0}(x)\right\|$ s'étend par continuité à tout $x \in A$. Le lemme résulte alors du lemme 2.1 de (3).

Lemme 2. Soient $A$ une $C^{*}$-algèbre, $B$ une partie partout dense de $A$, et $\mathscr{T}$ une topologie sur $\hat{A}$. On suppose que, pour tout $x \in B$, la fonction $T \rightarrow\|T(x)\|$ est continue pour $\mathscr{T}$. Alors $\mathscr{T}$ est plus fine que la topologie canonique de $A$.

Démonstration. Soit $S$ une partie canoniquement fermée de $\hat{A}$. Si $T_{0} \in \hat{A}$ est adhérent à $S$ pour $\mathscr{T}$, on a $\sup _{T \epsilon S}\|T(x)\| \geqslant\left\|T_{0}(x)\right\|$ pour tout $x \in B$. Donc (Lemme 1) $T_{0}$ est canoniquement adhérent à $S$ et par suite $T_{0} \in S$. Donc $S$ est fermée pour $\mathscr{T}$.

Lemme 3. Soient $\Gamma$ un groupe localement compact, $\Gamma^{\prime}$ un sous-groupe distingué fermé de $\Gamma$, et $\varphi$ l'application canonique de $\Gamma$ sur $\Gamma / \Gamma^{\prime}$. Alors l'application $\hat{\varphi}: U \rightarrow U \circ \varphi$ de $\left(\Gamma / \Gamma^{\prime}\right)^{-}$dans $\bar{\Gamma}$ est un homéomorphisme de $\left(\Gamma / \Gamma^{\prime}\right)^{-}$sur une partie fermée de $\bar{\Gamma}$.

Démonstration. Le fait que $\varphi$ soit un homéomorphisme de $\left(\Gamma / \Gamma^{\prime}\right)$ - sur une partie $S$ de $\bar{\Gamma}$ résulte aussitôt du Théorème 1.5 de (3) et du fait qu'une partie 
de $\Gamma / \Gamma^{\prime}$ est compacte si et seulement si elle est l'image par $\varphi$ d'une partie compacte de $\Gamma$. D'autre part, si $T \in \bar{\Gamma}$ est canoniquement adhérent à $S$, le Théorème 1.5 de (3) montre que les fonctions de type positif associées à $T$ sont constantes sur les classes modulo $\Gamma^{\prime}$. En particulier, si $x$ est un vecteur unitaire de l'espace où opère $T$, on a $(T(\gamma) x \mid x)=1$ pour $\gamma \in \Gamma^{\prime}$, donc $T(\gamma) x=x$ pour $\gamma \in \Gamma^{\prime}$, donc $T(\gamma)=1$ pour $\gamma \in \Gamma^{\prime}$. Donc $T \in S$.

Lemme 4. Soient $F \in \mathscr{S}\left(\mathbf{R}^{n}\right)$, $\Lambda$ un espace topologique, $\lambda_{0} \in \Lambda$, et $\varphi$ une application continue de $\mathbf{R}^{p} \times \Lambda$ dans $\mathbf{R}^{n}$ possédant la propriété suivante: il existe un $K>0$, un $\alpha>0$, et un voisinage $V$ de $\lambda_{0}$ dans $\Lambda$ tel que $\|\varphi(x, \lambda)\| \geqslant$ $K\|x\| \|^{\alpha}$ pour $\|x\| \geqslant 1$ et $\lambda \in V$. Posons $\psi_{\lambda}(x)=F(\varphi(x, \lambda))$. Alors, quand $\lambda \rightarrow \lambda_{0}$,

dans $L_{\mathbf{C}}{ }^{2}\left(\mathbf{R}^{p}\right)$.

$$
\psi_{\lambda} \rightarrow \psi_{\lambda_{0}}
$$

Démonstration. Soit $q=(p+1 /) 2 \alpha$. Il existe une constante $k>0$ telle que $|F(y)|\left(1+\|y\|^{q}\right) \leqslant k$. Alors, pour $\lambda \in V$ et $x \in \mathbf{R}^{p},\|x\| \geqslant 1$, on a

$\left|\psi_{\lambda}(x)\right|=|F(\varphi(x, \lambda))| \leqslant \frac{k}{1+\|\varphi(x, \lambda)\|^{q}} \leqslant \frac{k}{1+K^{q}|| x \|^{\alpha q}}=\frac{k}{1+K^{q}|| x \|^{\frac{1}{1}(\bar{p}+1)}}$.

Donc $\psi_{\lambda} \in L_{\mathbf{C}^{2}}\left(\mathbf{R}^{p}\right)$, et

$$
\left|\psi_{\lambda}-\psi_{\lambda_{0}}\right|
$$

est, pour $\lambda \in V$, majoré par une fonction fixe de $L_{\mathbf{G}^{2}}{ }^{2}\left(\mathbf{R}^{p}\right)$. Comme

$$
\psi_{\lambda}(x)-\psi_{\lambda_{0}}(x) \rightarrow 0
$$

pour chaque valeur de $x$ quand $\lambda \rightarrow \lambda_{0}$, on voit que

$$
\int\left|\psi_{\lambda}(x)-\psi_{\lambda_{0}}(x)\right|^{2} d x \rightarrow 0
$$

quand $\lambda \rightarrow \lambda_{0}$.

2. Topologie de $\bar{\Gamma}_{3}$. D'après (1), proposition $3, \bar{\Gamma}_{3}$ est réunion de 2 sous-ensembles disjoints $A$ et $B$ :

(1) $A$ est l'ensemble des représentations $U_{\lambda}(\lambda \in \mathbf{R}, \lambda \neq 0)$; chaque représentation $U_{\lambda}$ opère dans $L_{\mathbf{C}}{ }^{2}(\mathbf{R})$ et est définie par la formule

$$
\begin{aligned}
& \left(U_{\lambda}(\gamma) f\right)(\theta)=\left[\exp i \lambda\left(\rho_{3}-\rho_{2} \theta\right)\right] f\left(\theta+\rho_{1}\right) \\
& \left(\gamma=\left(\rho_{1}, \rho_{2}, \rho_{3}\right) \in \Gamma_{3}, \quad f \in L_{\mathbf{C}}{ }^{2}(\mathbf{R}), \quad \theta \in \mathbf{R}\right) .
\end{aligned}
$$

Toutes les fois que cela sera commode, nous identifierons $A$ et $\mathbf{R}-\{0\}$ par l'application $\lambda \rightarrow U_{\lambda}$.

(2) $B$ est l'ensemble des représentations $V_{\lambda, \mu}(\lambda, \mu \in \mathbf{R})$; chaque représentation $V_{\lambda, \mu}$ opère dans un espace de dimension 1 , donc s'identifie à une fonction scalaire sur $\Gamma_{3}$, conformément à la relation

$$
V_{\lambda, \mu}(\gamma)=\exp i\left(\lambda \rho_{1}+\mu \rho_{2}\right)
$$

$\left(\gamma=\left(\rho_{1}, \rho_{2}, \rho_{3}\right) \in \Gamma_{3}\right)$. Nous identifierons éventuellement $B$ et $\mathbf{R}^{2}$ par l'application $(\lambda, \mu) \rightarrow V_{\lambda, \mu}$. 
Munissons $A$ de la topologie de $\mathbf{R}-\{0\}$ et $B$ de la topologie de $\mathbf{R}^{2}$. La topologie sur $\bar{\Gamma}_{3}$ qui fait de $\bar{\Gamma}_{3}$ la somme topologique des espaces topologiques $A$ et $B$ précédents sera appelée la topologie des paramètres. D'après le Lemme 3 , $B$ est canoniquement fermé dans $\bar{\Gamma}_{3}$, et la topologie induite sur $B$ par la topologie canonique de $\bar{\Gamma}_{3}$ est la topologie des paramètres.

Soit $F \in \mathscr{S}\left(\Gamma_{3}\right)$. Soient $f$ et $g$ des fonctions numériques complexes d'une variable réelle, continues à support compact. On a

$$
\begin{aligned}
& \left(U_{\lambda}(F) f \mid g\right) \\
& \quad=\iiint F\left(\rho_{1}, \rho_{2}, \rho_{3}\right) d \rho_{1} d \rho_{2} d \rho_{3} \int \exp i \lambda\left(\rho_{3}-\rho_{2} \theta\right) f\left(\theta+\rho_{1}\right) \overline{g(\theta)} d \theta \\
& \quad=\iiint \int F\left(\rho_{1}-\theta, \rho_{2}, \rho_{3}\right) \exp i \lambda\left(\rho_{3}-\rho_{2} \theta\right) f\left(\rho_{1}\right) \overline{g(\theta)} d \rho_{1} d \rho_{2} d \rho_{3} d \theta .
\end{aligned}
$$

Donc $U_{\lambda}(F)$ est défini par le noyau

$$
\begin{aligned}
K_{\lambda}\left(\rho_{1}, \theta\right) & =\iint F\left(\rho_{1}-\theta, \rho_{2}, \rho_{3}\right) \exp i \lambda\left(\rho_{3}-\rho_{2} \theta\right) d \rho_{2} d \rho_{3} \\
& =\hat{F}_{23}\left(\rho_{1}-\theta, \lambda \theta,-\lambda\right) .
\end{aligned}
$$

Lemme 5. Soit $F \in \mathscr{S}\left(\Gamma_{3}\right)$. La fonction $T \rightarrow\|T(F)\|$ est continue sur $\bar{\Gamma}_{3}$ pour la topologie des paramètres.

Démonstration. La continuité est immédiate sur $B$. D'autre part, en utilisant les notations précédentes, l'application $\lambda \rightarrow K_{\lambda}$ est une application fortement continue de $\mathbf{R}-\{0\}$ dans $L_{\mathbf{C}}{ }^{2}\left(\mathbf{R}^{2}\right)$ (Lemme 4). Donc l'application $\lambda \rightarrow U_{\lambda}(F)$ est continue pour la norme d'Hilbert-Schmidt des opérateurs et a fortiori pour la topologie uniforme. Ceci prouve la continuité de l'application $\lambda \rightarrow\left\|U_{\lambda}(F)\right\|$ sur $A$. D'où le lemme.

Utilisant le Lemme 2, on voit que les ensembles canoniquement fermés de $\bar{\Gamma}_{3}$ sont à chercher parmi les ensembles $A_{1} \cup B_{1}$, où $A_{1} \subset A$ et $B_{1} \subset B$ sont fermés pour la topologie des paramètres. En outre, $A_{1}$ et $B_{1}$ doivent vérifier des conditions supplémentaires:

Lemme 6. Soient $A_{1} \subset A, B_{1} \subset B$. On suppose $A_{1} \cup B_{1}$ canoniquement fermé. Alors, si 0 est adhérent à $A_{1}$ dans $\mathbf{R}$, on a $B_{1}=B$.

Démonstration. Soit $F \in \mathscr{S}\left(\Gamma_{3}\right)$. Soient $f$ et $g$ des fonctions numériques complexes d'une variable réelle, continues à support compact. Pour tout $\beta \in \mathbf{R}$, on a, d'après (1)

$$
\begin{aligned}
\left(U_{\lambda}(F)\right. & \left.\mathscr{T}\left(\frac{\beta}{\lambda}\right) f \mid \mathscr{T}\left(\frac{\beta}{\lambda}\right) g\right) \\
& =\iiint \int F\left(\rho_{1}, \rho_{2}, \rho_{3}\right) \exp i \lambda\left(\rho_{3}-\rho_{2} \theta\right) f\left(\theta+\rho_{1}+\frac{\beta}{\lambda}\right) \overline{\left(\theta+\frac{\beta}{\lambda}\right)} d \rho_{1} d \rho_{2} d \rho_{3} d \theta \\
& =\iiint \int F\left(\rho_{1}, \rho_{2}, \rho_{3}\right) \exp i\left(\lambda \rho_{3}-\lambda \rho_{2} \theta+\beta \rho_{2}\right) f\left(\theta+\rho_{1}\right) \overline{g(\theta)} d \rho_{1} d \rho_{2} d \rho_{3} d \theta
\end{aligned}
$$

Quand $\lambda \rightarrow 0$, ceci tend vers 


$$
\begin{aligned}
\iiint \int F\left(\rho_{1}, \rho_{2}, \rho_{3}\right) \exp \left(i \beta \rho_{2}\right) f(\theta & \left.+\rho_{1}\right) \overline{g(\theta)} d \rho_{1} d \rho_{2} d \rho_{3} d \theta \\
& =\iint \hat{F}_{23}\left(\rho_{1},-\beta, 0\right) f\left(\theta+\rho_{1}\right) \overline{g(\theta)} d \rho_{1} d \theta=(V f \mid g),
\end{aligned}
$$

où $V$ est, dans $L_{\mathbf{C}^{2}}(\mathbf{R})$, l'opérateur de convolution avec la fonction $\rho_{1} \rightarrow$ $\hat{F}_{23}\left(-\rho_{1},-\beta, 0\right)$. Ainsi, quand $\lambda \rightarrow 0$,

$$
\mathscr{T}\left(\frac{\beta}{\lambda}\right)^{-1} U_{\lambda}(F) \mathscr{T}\left(\frac{\beta}{\lambda}\right)
$$

tend faiblement vers $V$, donc $\lim _{\lambda_{\lambda \rightarrow 0}}\left\|U_{\lambda}(F)\right\| \geqslant\|V\|$. Par transformation de Fourier, on voit que

$$
\|V\|=\sup _{\xi \in \mathbf{R}}\left|\hat{F}_{123}(\xi,-\beta, 0)\right|=\sup _{\xi \in \mathbf{R}}|| V_{-\xi, \beta}(F) \| .
$$

Donc $\underline{\lim }_{\lambda \rightarrow 0}\left\|U_{\lambda}(F)\right\| \geqslant\left\|V_{\xi, \mu}(F)\right\|$ quels que soient $\xi \in \mathbf{R}, \mu \in \mathbf{R}$. Le Lemme 6 résulte alors du Lemme 1 .

Proposition 1. Les ensembles canoniquement fermés dans $\bar{\Gamma}_{3}$ sont les ensembles $A_{1} \cup B_{1}\left(A_{1} \subset A, B_{1} \subset B\right)$ possédant les propriétés suivantes:

(1) $A_{1}$ est fermé dans $A$ pour la topologie des paramètres;

(2) $B_{1}$ est fermé dans $B$ pour la topologie des paramètres;

(3) Si 0 est adhérent à $A_{1}$ dans $\mathbf{R}$, on a $B_{1}=B$.

Démonstration. Nous savons déjà que les conditions (1), (2), et (3) sont nécessaires pour que $A_{1} \cup B_{1}$ soit canoniquement fermé. Supposons ces conditions remplies et montrons que $A_{1} \cup B_{1}$ est canoniquement fermé. Soit $T \in \bar{\Gamma}_{3}, T \notin A_{1} \cup B_{1}$. Il s'agit de montrer que $T$ n'est pas canoniquement adhérent à $A_{1} \cup B_{1}$. Or $T$ n'est pas canoniquement adhérent à $B_{1}$ car $B_{1}$ est canoniquement fermé. Il s'agit donc de prouver que $T$ n'est pas canoniquement adhérent à $A_{1}$. Nous allons pour cela construire une $F \in \mathscr{S}\left(\Gamma_{3}\right)$ telle que $T(F) \neq 0$, et telle que $T^{\prime}(F)=0$ pour $T^{\prime} \in A_{1}$. Distinguons deux cas.

$$
T=U_{\lambda_{0}} \notin A_{1} .
$$

Soient $\theta \in \mathbf{R}, \rho_{1} \in \mathbf{R}$. Soit $E$ l'ensemble des points de $\mathbf{R}^{3}$ dont la 3 e coordonnée appartient à $-A_{1}$. Puisque $A_{1}$ est fermé pour la topologie des paramètres, le point $\left(\rho_{1}-\theta, \lambda_{0} \theta,-\lambda_{0}\right)$ est extérieur à $E$. Soit $G$ une fonction de $\mathscr{S}\left(\mathbf{R}^{3}\right)$ non nulle en ce point et nulle sur $E$. On a $G=\hat{F}_{23}$ pour $F \in \mathscr{S}\left(\mathbf{R}^{3}\right)$ bien choisie. Alors, compte tenu de $(2), U_{\lambda_{0}}(F)$ est défini par un noyau de $\mathscr{S}\left(\mathbf{R}^{3}\right)$ qui n'est pas identiquement nul, donc

$$
U_{\lambda_{0}}(F) \neq 0
$$

par contre, pour $\lambda \in A_{1}, U_{\lambda}(F)$ est défini par le noyau 0 , donc $U_{\lambda}(F)=0$.

$$
T=V_{\lambda_{0}, \mu_{0}} \notin B_{1} .
$$

D'après l'hypothèse (3) de la proposition, 0 n'est pas adhérent à $A_{1}$ dans 
R. Le point $\left(-\lambda_{0},-\mu_{0}, 0\right)$ est donc extérieur à l'ensemble $E$ introduit plus haut. Il existe une $F \in \mathscr{S}\left(\mathbf{R}^{3}\right)$ telle que $U_{\lambda}(F)=0$ pour $\lambda \in A_{1}$, et $\hat{F}_{123}\left(-\lambda_{0},-\mu_{0}, 0\right) \neq 0$, donc

$$
V_{\lambda_{0}, \mu_{0}}(F) \neq 0 .
$$

Remarque. Le quotient de $\Gamma_{3}$ par un sous-groupe discret non trivial de son centre a donc un dual localement compact, qui s'identifie à la somme topologique de $\mathbf{R}^{2}$ et de $\mathbf{Z}-\{0\}$ ( $\mathbf{Z}$ : ensemble des entiers rationnels).

3. Topologie de $\bar{\Gamma}_{4}$. D'après (1), Proposition $4, \bar{\Gamma}_{4}$ est réunion de 3 sousensembles disjoints $A, B, C$ :

(1) $A$ est l'ensemble des $U_{\lambda, \mu}(\lambda, \mu \in \mathbf{R}, \lambda \neq 0)$; chaque $U_{\lambda, \mu}$ opère dans $L_{\mathbf{G}}{ }^{2}(\mathbf{R})$ et est définie par la formule

$$
\begin{aligned}
\left(U_{\lambda, \mu}(\gamma) f\right)(\theta) & =\exp i\left(-\frac{1}{2} \frac{\mu}{\lambda} \rho_{2}+\lambda \rho_{4}-\lambda \rho_{3} \theta+\frac{1}{2} \lambda \rho_{2} \theta^{2}\right) f\left(\theta+\rho_{1}\right) \\
(\gamma & \left.=\left(\rho_{1}, \rho_{2}, \rho_{3}, \rho_{4}\right) \in \Gamma_{4}, f \in L_{\mathbf{C}}^{2}(\mathbf{R}), \theta \in \mathbf{R}\right) .
\end{aligned}
$$

(2) $B$ est l'ensemble des $V_{\lambda}(\lambda \in \mathbf{R}, \lambda \neq 0)$; chaque $V_{\lambda}$ opère dans $L_{\mathbf{C}}{ }^{2}(\mathbf{R})$ et est définie par la formule

$$
\left(V_{\lambda}(\gamma) f\right)(\theta)=\exp i \lambda\left(\rho_{3}-\rho_{2} \theta\right) f\left(\theta+\rho_{1}\right) .
$$

(3) $C$ est l'ensemble des $W_{\lambda, \mu}(\lambda, \mu \in \mathbf{R})$; chaque $W_{\lambda, \mu}$ s'identifie à une fonction scalaire sur $\Gamma_{4}$ conformément à la relation

$$
W_{\lambda, \mu}(\gamma)=\exp i\left(\lambda \rho_{1}+\mu \rho_{2}\right) .
$$

D'après le Lemme $3, B \cup C$ est canoniquement fermé dans $\bar{\Gamma}_{4}$, et la topologie induite sur $B \cup C$ par la topologie canonique de $\bar{\Gamma}_{4}$ s'identifie à la topologie canonique de $\bar{\Gamma}_{3}$; elle est donc connue par la Proposition 1.

On définit sur $\bar{\Gamma}_{4}$ une "topologie des paramètres" comme on l'a fait sur $\bar{\Gamma}_{3}$.

Nous noterons $\Pi_{+}$(resp. II_) l'ensemble des $(\lambda, \mu) \in \mathbf{R}^{2}$ tels que $\lambda>0$ (resp. $\lambda<0$ ). Nous identifierons $A$ à $\Pi_{+} \cup \Pi_{-}, B$ à $\mathbf{R}-\{0\}$ et $C$ à $\mathbf{R}^{2}$ quand cela sera commode.

Soit $F \in \mathscr{S}\left(\Gamma_{4}\right)$. Soient $f$ et $g$ deux fonctions numériques complexes d'une variable réelle, continues à support compact. On a

(3) $\quad\left(U_{\lambda, \mu}(F) f \mid g\right)$

$$
\begin{array}{r}
=\int \ldots \int F\left(\rho_{1}, \ldots, \rho_{4}\right) \exp i\left(-\frac{1}{2} \frac{\mu}{\lambda} \rho_{2}+\lambda \rho_{4}-\lambda \rho_{3} \theta+\frac{1}{2} \lambda \rho_{2} \theta^{2}\right) f\left(\theta+\rho_{1}\right) \overline{g(\theta)} \\
d \rho_{1} \ldots d \rho_{4} d \theta \\
=\int \ldots \int F\left(\rho_{1}-\theta, \rho_{2}, \rho_{3}, \rho_{4}\right) \exp i\left(-\frac{1}{2} \frac{\mu}{\lambda} \rho_{2}+\lambda \rho_{4}-\lambda \rho_{3} \theta+\frac{1}{2} \lambda \rho_{2} \theta^{2}\right) \\
f\left(\rho_{1}\right) \overline{g(\theta)} d \rho_{1} \ldots d \rho_{4} d \theta .
\end{array}
$$


Donc $U_{\lambda, \mu}(F)$ est défini par le noyau

(4) $K_{\lambda, \mu}\left(\rho_{1}, \theta\right)$

$$
\begin{aligned}
& =\iiint F\left(\rho_{1}-\theta, \rho_{2}, \rho_{3}, \rho_{4}\right) \exp i\left(-\frac{1}{2} \frac{\mu}{\lambda} \rho_{2}+\lambda \rho_{4}-\lambda \rho_{3} \theta+\frac{1}{2} \lambda \rho_{2} \theta^{2}\right) d \rho_{2} d \rho_{3} d \rho_{4} \\
& =\hat{F}_{234}\left(\rho_{1}-\theta, \frac{1}{2} \frac{\mu}{\lambda}-\frac{1}{2} \lambda \theta^{2}, \lambda \theta,-\lambda\right) .
\end{aligned}
$$

Lemme 7. Soit $F \in \mathscr{P}\left(\Gamma_{4}\right)$. La fonction $T \rightarrow\|T(F)\|$ est continue sur $\bar{\Gamma}_{4}$ pour la topologie des paramètres.

Démonstration. La continuité sur $B \cup C$ résulte du Lemme 5. D'autre part, en utilisant les notations précédentes, l'application $(\lambda, \mu) \rightarrow K_{\lambda, \mu}$ est une application fortement continue de $A$ dans $L_{\mathbf{G}}{ }^{2}\left(\mathbf{R}^{2}\right)$ (Lemme 4 ). On en conclut comme au Lemme 5 que l'application $(\lambda, \mu) \rightarrow\left\|U_{\lambda, \mu}(F)\right\|$ est continue sur $A$.

Lemme 8. Soient $A_{1} \subset A, B_{1} \subset B, C_{1} \subset C$. On suppose $A_{1} \cup B_{1} \cup C_{1}$ canoniquement fermé. Alors:

(1) Si $\mu_{0}>0$ est tel que $\left(0, \mu_{0}\right)$ soit adhérent à $A_{1}$ dans $\mathbf{R}^{2}$, on a

$$
V \mu_{0}^{\frac{1}{2}} \in B_{1}, V-\mu_{0}^{\frac{1}{2}} \in B_{1} .
$$

(2) Si $(0,0)$ est adhérent à $A_{1} \cap \mathrm{I}^{+}$dans $\mathbf{R}^{2}$, on a $W_{\lambda_{0}, \mu_{0}} \in C_{1}$ pour

$$
\mu_{0} \geqslant \underline{\lim }_{(\lambda, \mu)_{\rightarrow}(0,0),(\lambda ; \mu) \epsilon A_{1} \cap \mathrm{II}_{+}}\left(-\frac{\mu}{2 \lambda}\right) .
$$

(3) Si $(0,0)$ est adhérent à $A_{1} \cap \Pi_{-}$dans $\mathbf{R}^{2}$, on a $W_{\lambda_{0}, \mu_{0}} \in C_{1}$ pour

$$
\mu_{0} \leqslant \overline{\lim }_{(\lambda, \mu) \rightarrow(0,0),(\lambda, \mu) \epsilon_{1} \cap \Pi-}\left(-\frac{1}{2} \frac{\mu}{\lambda}\right) .
$$

Démonstration. Soit $F \in \mathscr{S}\left(\Gamma_{4}\right)$. Soient $f$ et $g$ des fonctions numériques complexes d'une variable réelle, continues à support compact. Pour $\mu \geqslant 0$, on a, d'après (3)

$$
\begin{aligned}
& \left(U_{\lambda, \mu}(F) \mathscr{T}\left(\frac{\mu^{\frac{1}{2}}}{\lambda}\right) f \mid \mathscr{T}\left(\frac{\mu^{\frac{1}{2}}}{\lambda}\right) g\right)=\int \ldots \int F\left(\rho_{1}, \ldots, \rho_{4}\right) \exp (i \Delta) f\left(\theta+\rho_{1}\right) \\
& \overline{g(\theta)} d \rho_{1} \ldots d \rho_{4} d \theta
\end{aligned}
$$

avec

$$
\begin{aligned}
\Delta & =-\frac{1}{2} \frac{\mu}{\lambda} \rho_{2}+\lambda \rho_{4}-\lambda \rho_{3}\left(\theta-\frac{\mu^{\frac{1}{2}}}{\lambda}\right)+\frac{1}{2} \lambda \rho_{2}\left(\theta-\frac{\mu^{\frac{1}{2}}}{\lambda}\right)^{2} \\
& =\lambda \rho_{4}-\lambda \rho_{3} \theta+\mu^{\frac{1}{2}} \rho_{3}+\frac{1}{2} \lambda \rho_{2} \theta^{2}-\mu^{\frac{1}{2}} \rho_{2} \theta
\end{aligned}
$$


Quand $\lambda \rightarrow 0$ et $\mu \rightarrow \mu_{0}>0$, l'intégrale tend vers

$$
\begin{aligned}
\int \ldots \int F\left(\rho_{1}, \ldots, \rho_{4}\right) \exp i \mu_{0}^{\frac{1}{2}}\left(\rho_{3}-\rho_{2} \theta\right) f\left(\theta+\rho_{1}\right) \overline{g(\theta)} d \rho_{1} \ldots d \rho_{4} d \theta \\
=\left(V \mu_{0}^{\frac{1}{2}}(F) f \mid g\right) .
\end{aligned}
$$

Donc $\mathscr{T}\left(\mu^{\frac{1}{2}} / \lambda\right)^{-1} U_{\lambda, \mu}(F) \mathscr{T}\left(\mu^{\frac{1}{2}} / \lambda\right)$ tend faiblement vers $V_{\mu_{0}^{\frac{1}{2}}}$, et par suite

$$
\underline{\lim }_{(\lambda, \mu) \rightarrow\left(0, \mu_{0}\right)}\left\|U_{\lambda, \mu}(F)\right\| \geqslant\left\|V_{0}^{\frac{1}{2}}\right\|
$$

Si $\left(0, \mu_{0}\right)$ est adhérent à $A_{1}$ dans $\mathbf{R}^{2}$, on a

$$
V_{\mu_{0}^{2}}^{\frac{1}{2}} \in B_{1}
$$

(Lemme 1). Considérant $\mathscr{T}\left(-\mu^{\frac{1}{2}} / \lambda\right)^{-1} U_{\lambda, \mu}(F) \mathscr{T}\left(-\mu^{\frac{1}{2}} / \lambda\right)$, on voit de même

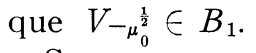

Supposons maintenant que $(0,0)$ soit adhérent à $A_{1} \cap \Pi_{+}$dans $\mathbf{R}^{2}$. Soit $\mu_{0}$ un nombre réel fini tel que

$$
\mu_{0} \geqslant \underline{\lim }_{(\lambda, \mu) \rightarrow(0,0),(\lambda, \mu) \epsilon A_{1} \cap \Pi_{+}}\left(-\frac{1}{2} \frac{\mu}{\lambda}\right) .
$$

Il existe des suites de nombres réels $\lambda_{n}, \mu_{n}, \beta_{n}$ tels que

$$
\left(\lambda_{n}, \mu_{n}\right) \rightarrow(0,0),\left(\lambda_{n}, \mu_{n}\right) \in A_{1} \cap \mathrm{II}_{+},-\frac{1}{2} \frac{\mu_{n}}{\lambda_{n}}+\frac{1}{2} \lambda_{n} \beta_{n}^{2} \rightarrow \mu_{0}
$$

Alors,

$$
\begin{aligned}
\left(U_{\lambda_{n}, \mu_{n}}(F) \mathscr{T}\left(\beta_{n}\right) f \mid \mathscr{T}\left(\beta_{n}\right) g\right) & \\
& =\int \ldots \int F\left(\rho_{1}, \ldots, \rho_{4}\right) \exp (i \Delta) f\left(\theta+\rho_{1}\right) \overline{g(\theta)} d \rho_{1} \ldots d \rho_{4} d \theta
\end{aligned}
$$

avec

$$
\begin{aligned}
\Delta & =-\frac{1}{2} \frac{\mu_{n}}{\lambda_{n}} \rho_{2}+\lambda_{n} \rho_{4}-\lambda_{n} \rho_{3}\left(\theta-\beta_{n}\right)+\frac{1}{2} \lambda_{n} \rho_{2}\left(\theta-\beta_{n}\right)^{2} \\
& =\left(-\frac{1}{2} \frac{\mu_{n}}{\lambda_{n}}+\frac{1}{2} \lambda_{n} \beta_{n}^{2}\right) \rho_{2}+\lambda_{n} \rho_{4}-\lambda_{n} \rho_{3} \theta+\frac{1}{2} \lambda_{n} \rho_{2} \theta^{2}+\lambda_{n} \beta_{n}\left(\rho_{3}-\rho_{2} \theta\right)
\end{aligned}
$$

Remarquons que $-\frac{1}{2} \mu_{n}+\frac{1}{2} \lambda_{n}{ }^{2} \beta_{n}{ }^{2} \rightarrow 0$, donc que $\lambda_{n} \beta_{n} \rightarrow 0$, quand $n \rightarrow+\infty$. Ceci posé, l'intégrale tend vers

$$
\begin{aligned}
\int \ldots \int F\left(\rho_{1}, \ldots, \rho_{4}\right) & \exp \left(i \mu_{0} \rho_{2}\right) f\left(\theta+\rho_{1}\right) \overline{g(\theta)} d \rho_{1} \ldots d \rho_{4} d \theta \\
& =\iint \hat{F}_{234}\left(\rho_{1},-\mu_{0}, 0,0\right) f\left(\theta+\rho_{1}\right) \overline{g(\theta)} d \rho_{1} d \theta=(V f \mid g)
\end{aligned}
$$

où $V$ est, dans $L_{\mathbf{C}^{2}}(\mathbf{R})$, l'opérateur de convolution avec la fonction $\rho_{1} \rightarrow$ $\hat{F}_{234}\left(-\rho_{1},-\mu_{0}, 0,0\right)$. Quand $n \rightarrow+\infty$,

$$
\mathscr{T}\left(\beta_{n}\right)^{-1} U_{\lambda_{n}, \mu_{n}}(F) \mathscr{T}\left(\beta_{n}\right)
$$


tend faiblement vers $V$, donc

$$
\underline{\lim }_{n \rightarrow+\infty}|| U_{\lambda_{n}, \mu_{n}}(F)\left\|\geqslant \sup _{\xi \in \mathbf{R}}\left|\hat{F}_{1234}\left(-\xi,-\mu_{0}, 0,0\right)\right|=\sup _{\xi \in \mathbf{R}}|| W_{\xi, \mu_{0}}(F)\right\| .
$$

Donc $W_{\xi, \mu_{0}} \in C_{1}$ pour tout $\xi \in \mathbf{R}$. On raisonne de façon analogue lorsque $(0,0)$ est adhérent à $A_{1} \cap \Pi_{-}$dans $\mathbf{R}^{2}$.

Le Lemme 8 donne des conditions nécessaires pour que $\Lambda_{1} \cup B_{1} \cup C_{1}$ soit canoniquement fermé. Pour prouver que ces conditions sont aussi suffisantes, nous aurons besoin du lemme suivant.

Lemme 9. Soit $A_{1}$ une partie de A fermée pour la topologie des paramètres. Soit E l'ensemble des points de $\mathbf{R}^{4}$ de la forme

$$
\left(t, \frac{1}{2} \frac{\mu}{\lambda}-\frac{1}{2} \lambda \theta^{2}, \lambda \theta,-\lambda\right)
$$

où $t \in \mathbf{R}, \theta \in \mathbf{R},(\lambda, \mu) \in A_{1}$. Alors l'adhérence de $E$ dans $\mathbf{R}^{4}$ est contenue dans la réunion des ensembles suivants:

(1) $E$;

(2) l'ensemble des points $\left(t, \tau, \pm \mu_{0}^{\frac{1}{2}}, 0\right)$, où $t \in \mathbf{R}, \tau \in \mathbf{R}$, et où $\mu_{0}>0$ est tel que $\left(0, \mu_{0}\right)$ soit adhérent à $A_{1}$ dans $\mathbf{R}^{2}$;

(3) si $(0,0)$ est adhérent à $A_{1} \cap \Pi_{+}$dans $\mathbf{R}^{2}$, l'ensemble des points $(t, \tau, 0,0)$, où

$$
\tau \leqslant \overline{\lim }_{(\lambda, \mu) \rightarrow(0,0),(\lambda, \mu) \epsilon A_{1} \cap \Pi_{+}}\left(\frac{1}{2} \frac{\mu}{\lambda}\right) ;
$$

(t) si $(0,0)$ est adhérent à $A_{1} \cap \Pi_{-}$dans $\mathbf{R}^{2}$, l'ensemble des points $(t, \tau, 0,0)$, où

$$
\tau \geqslant \underline{\lim }_{(\lambda, \mu)_{\rightarrow}(0,0),(\lambda, \mu) \in A_{1} \cap \Pi-}\left(\frac{1}{2} \frac{\mu}{\lambda}\right) .
$$

(En fait, l'énoncé précédent fournit exactement l'adhérence de $E$; mais nous n'aurons pas besoin de ce fait.)

Démonstration. Supposons que

$$
t_{n} \rightarrow \xi_{1}, \frac{1}{2} \frac{\mu_{n}}{\lambda_{n}}-\frac{1}{2} \lambda_{n} \theta_{n}^{2} \rightarrow \xi_{2}, \lambda_{n} \theta_{n} \rightarrow \xi_{3},-\lambda_{n} \rightarrow \xi_{4}
$$

$\left(t_{n} \in \mathbf{R}, \theta_{n} \in \mathbf{R},\left(\lambda_{n}, \mu_{n}\right) \in A_{1}\right)$, et montrons que $\left(\xi_{1}, \xi_{2}, \xi_{3}, \xi_{4}\right)$ appartient à l'un des ensembles du lemme. Si $\xi_{4} \neq 0, t_{n}, \theta_{n}, \lambda_{n}, \mu_{n}$ ont des limites finies $t, \theta, \lambda, \mu$; on a $(\lambda, \mu) \in A_{1}$ puisque $A_{1}$ est fermé dans $A$ pour la topologie des paramètres, et

$$
\xi_{1}=t, \xi_{2}=\frac{1}{2} \frac{\mu}{\lambda}-\frac{1}{2} \lambda \theta^{2}, \xi_{3}=\lambda \theta, \xi_{4}=-\lambda,
$$

donc $\left(\xi_{1}, \xi_{2}, \xi_{3}, \xi_{4}\right) \in E$. Supposons $\xi_{4}=0$. Comme $\lambda_{n}{ }^{-1}\left(\mu_{n}-\lambda_{n}{ }^{2} \theta_{n}{ }^{2}\right) \rightarrow 2 \xi_{2}$, on a $\mu_{n}-\lambda_{n}{ }^{2} \theta_{n}{ }^{2} \rightarrow 0$, donc $\mu_{n} \rightarrow \xi_{3}{ }^{2}$. Si $\xi_{3} \neq 0$, alors $\mu_{0}=\xi_{3}{ }^{2}>0,\left(0, \mu_{0}\right)$ est 
adhérent à $A_{1}$ dans $\mathbf{R}^{2}$, et $\left(\xi_{1}, \xi_{2}, \xi_{3}, \xi_{4}\right)=\left(\xi_{1}, \xi_{2}, \pm \mu_{0}^{\frac{1}{2}}, 0\right)$. Enfin, si $\xi_{3}=\xi_{4}=0$, $(0,0)$ est adhérent à $A_{1}$ dans $\mathbf{R}^{2}$. Supposons $\lambda_{n}>0$ pour une infinité de valeurs de $n$ (donc, en changeant de notations, pour toute valeur de $n$ ). Alors, $(0,0)$ est adhérent à $A_{1} \cap \Pi_{+}$dans $\mathbf{R}^{2}$. On a

$$
\xi_{2}=\lim \left(\frac{1}{2} \frac{\mu_{n}}{\lambda_{n}}-\frac{1}{2} \lambda_{n} \theta_{n}^{2}\right) \leqslant \varlimsup\left(\frac{1}{2} \frac{\mu_{n}}{\lambda_{n}}\right) \leqslant \varlimsup_{(\lambda, \mu) \rightarrow(0,0),(\lambda, \mu) \epsilon A_{1} \cap \Pi_{+}}\left(\frac{1}{2} \frac{\mu}{\lambda}\right) .
$$

On raisonne de même si $\lambda_{n}<0$ pour une infinité de valeurs de $n$.

Proposition 2. Les ensembles canoniquement fermés dans $\bar{\Gamma}_{4}$ sont les ensembles $A_{1} \cup B_{1} \cup C_{1}\left(A_{1} \subset A, B_{1} \subset B, C_{1} \subset C\right)$ possédant les propriétés suivantes:

(1) $A_{1}$ est fermé dans $A$ pour la topologie des paramètres;

(2) $B_{1} \cup C_{1}$ est canoniquement fermé dans $B \cup C=\bar{\Gamma}_{3}$;

(3) Si $\left(0, \mu_{0}\right)$ (où $\mu_{0}>0$ ) est adhérent à $A_{1}$ dans $\mathbf{R}^{2}$, on a

$$
V_{ \pm}^{\mu_{0}^{\frac{1}{2}}} \in B_{1}
$$

(4) Si $(0,0)$ est adhérent à $A_{1} \cap \Pi_{+}$dans $\mathbf{R}^{2}$, on a

$$
W_{\lambda_{0}, \mu_{0}} \in C_{1}
$$

toutes les fois que

$$
\mu_{0} \geqslant \underline{\lim }_{(\lambda, \mu) \rightarrow(0,0),(\lambda, \mu) \epsilon A_{1} \cap \Pi_{+}}\left(-\frac{1}{2} \frac{\mu}{\lambda}\right) ;
$$

(5) Si $(0,0)$ est adhérent à $A_{1} \cap \Pi_{-}$dans $\mathbf{R}^{2}$, on a

$$
W_{\lambda_{0}, \mu_{0}} \in C_{1}
$$

toutes les fois que

$$
\mu_{0} \leqslant \overline{\lim }_{(\lambda, \mu) \rightarrow(0,0),(\lambda, \mu) \epsilon A_{1} \cap \Pi_{-}}\left(-\frac{1}{2} \frac{\mu}{\lambda}\right) .
$$

Démonstration. Raisonnant comme pour la Proposition 1, on est ramené à ceci: supposons vérifiées les conditions (1) à (5) de la proposition; soit $T \in \bar{\Gamma}_{4}, \quad T \notin A_{1} \cup B_{1} \cup C_{1}$; et montrons qu'il existe une $F \in \mathscr{S}\left(\Gamma_{4}\right)$ telle que $T(F) \neq 0$ et $T^{\prime}(F)=0$ pour $T^{\prime} \in A_{1}$. Distinguons trois cas.

$$
T=U_{\lambda_{0}, \mu_{0}} \notin A_{1} .
$$

Soient $\rho_{1} \in \mathbf{R}, \theta \in \mathbf{R}$. Utilisons le Lemme 9 et la notation $E$ de ce lemme. Le point

$$
\left(\rho_{1}-\theta, \frac{1}{2} \frac{\mu_{0}}{\lambda_{0}}-\frac{1}{2} \lambda_{0} \theta^{2}, \lambda_{0} \theta,-\lambda_{0}\right)
$$

n'appartient pas à $E$ (c'est immédiat), donc lui est extérieur (Lemme 9). Soit $G$ une fonction de $\mathscr{S}\left(\mathbf{R}^{4}\right)$ non nulle en ce point et nulle sur $E$. On a $G=\hat{F}_{234}$ pour $F \in \mathscr{S}\left(\mathbf{R}^{4}\right)$ bien choisie. Alors, compte tenu de (4), on a 


$$
U_{\lambda_{0}, \mu_{0}}(F) \neq 0,
$$

et $U_{\lambda, \mu}(F)=0$ pour $(\lambda, \mu) \in A_{1}$.

$$
T=V_{\lambda_{0}} \notin B_{1} .
$$

D'après l'hypothèse $(3)$ de la proposition, $\left(0, \lambda_{0}{ }^{2}\right)$ n'est pas adhérent à $A_{1}$ dans $\mathbf{R}^{2}$. D'après le Lemme 9 , quels que soient $\rho_{1} \in \mathbf{R}, \theta \in \mathbf{R}$, le point $\left(\rho_{1}-\theta, \lambda_{0} \theta,-\lambda_{0}, 0\right)$ est extérieur à $E$. Raisonnant comme dans (A), et compte tenu de (2) et (4), il existe $F \in \mathscr{P}\left(\mathbf{R}^{4}\right)$ telle que

$$
V_{\lambda_{0}}(F) \neq 0
$$

et $U_{\lambda, \mu}(F)=0$ pour $(\lambda, \mu) \in A_{1}$.

(C) $T=W_{\lambda_{0}, \mu_{0}} \notin C_{1}$. D'après les hypothèses (4) et (5) de la proposition, et compte tenu du Lemme $9,\left(-\lambda_{0},-\mu_{0}, 0,0\right)$ est extérieur à $E$. Raisonnant comme dans (A), il existe $F \in \mathscr{S}\left(\mathbf{R}^{4}\right)$ telle que $\hat{F}_{1234}$ soit nulle sur $E$ et non nulle en $\left(-\lambda_{0},-\mu_{0}, 0,0\right)$. Alors $\hat{F}_{234}$ est nulle sur $E$, donc $U_{\lambda, \mu}(F)=0$ pour $(\lambda, \mu) \in A_{1}$ et

$$
W_{\lambda_{0}, \mu_{0}}(F) \neq 0 \text {. }
$$

4. Topologie de $\bar{\Gamma}_{5,1}$. D'après (1, Proposition 5), $\bar{\Gamma}_{5,1}$ est une réunion de 2 sous-ensembles disjoints $A$ et $B$.

(1) $A$ est l'ensemble des $U_{\lambda}(\lambda \in \mathbf{R}, \lambda \neq 0)$; chaque $U_{\lambda}$ opère dans $\left.L_{\mathbf{C}^{2}}{ }^{2} \mathbf{R}^{2}\right)$ et est définie par la formule

$$
\begin{aligned}
\left(U_{\lambda}(\gamma) f\right)\left(\theta_{1}, \theta_{2}\right) & =\exp i \lambda\left(\rho_{5}-\rho_{2} \theta_{1}-\rho_{4} \theta_{2}\right) f\left(\theta_{1}+\rho_{1}, \theta_{2}+\rho_{3}\right) \\
(\gamma & \left.=\left(\rho_{1}, \ldots, \rho_{5}\right) \in \Gamma_{5,1}, f \in L_{\mathbf{C}}^{2}\left(\mathbf{R}^{2}\right), \theta_{1}, \theta_{2} \in \mathbf{R}\right) .
\end{aligned}
$$

(2) $B$ est l'ensemble des $V_{\lambda, \mu, \nu, \tau}(\lambda, \mu, \nu, \tau \in \mathbf{R})$; chaque $V_{\lambda, \mu, \nu, \tau}$ s'identifie à une fonction scalaire sur $\Gamma_{5,1}$, conformément à la relation

$$
V_{\lambda, \mu, \nu, \tau}(\gamma)=\exp i\left(\lambda \rho_{1}+\mu \rho_{2}+\nu \rho_{3}+\tau \rho_{4}\right) .
$$

D'après le Lemme $3, B$ est canoniquement fermé dans $\bar{\Gamma}_{5,1}$, et la topologie induite sur $B$ par la topologie canonique de $\bar{\Gamma}_{5,1}$ est la topologie canonique de $\mathbf{R}^{4}=\mathbf{R}^{4}$.

On définit sur $\bar{\Gamma}_{5,1}$ une "topologie des paramètres" comme on l'a fait sur $\bar{\Gamma}_{3}$. Ceci posé, les calculs du paragraphe 2 se transposent sans difficultés et fournissent le résultat suivant:

Proposition 3. Les ensembles canoniquement fermés dans $\bar{\Gamma}_{5,1}$ sont les ensembles $A_{1} \cup B_{1}\left(A_{1} \subset A, B_{1} \subset B\right)$ possédant les propriétés suivantes:

(1) $A_{1}$ est fermé dans $A$ pour la topologie des paramètres;

(2) $B_{1}$ est fermé dans $B$ pour la topologie des paramètres;

(3) Si 0 est adhérent à $A_{1}$ dans $\mathbf{R}$, on a $B_{1}=B$.

5. Topologie de $\bar{\Gamma}_{5,2}$ • D'après (1, Proposition 6 ), $\bar{\Gamma}_{E, 2}$ est réunion de 2 sous-ensembles disjoints $A, B$ : 
(1) $A$ est l'ensemble des $U_{\lambda, \mu, \nu}\left(\lambda, \mu, \nu \in \mathbf{R}, \lambda^{2}+\mu^{2} \neq 0\right)$; chaque $U_{\lambda, \mu, \nu}$ opère dans $L_{\mathbf{C}}{ }^{2}(\mathbf{R})$ et est définie par la formule

$$
\begin{aligned}
\left(U_{\lambda, \mu, \nu}(\gamma) f\right)(\theta) & =\exp i\left[\nu \frac{\lambda \rho_{3}-\mu \rho_{2}}{\lambda^{2}+\mu^{2}}+\lambda\left(\rho_{4}-\rho_{2} \theta\right)+\mu\left(\rho_{5}-\rho_{3} \theta\right)\right] f\left(\theta+\rho_{1}\right) \\
(\gamma & \left.=\left(\rho_{1}, \ldots, \rho_{5}\right) \in \Gamma_{5,1}, f \in L_{\mathbf{C}}^{2}(\mathbf{R}), \theta \in \mathbf{R}\right) .
\end{aligned}
$$

(2) $B$ est l'ensemble des $V_{\lambda, \mu, \nu}(\lambda, \mu, \nu \in \mathbf{R})$; chaque $V_{\lambda, \mu, \nu}$ s'identifie à une fonction scalaire sur $\Gamma_{5,2}$, conformément à la relation

$$
V_{\lambda, \mu, \nu}(\gamma)=\exp i\left(\lambda \rho_{1}+\mu \rho_{2}+\nu \rho_{3}\right) .
$$

D'après le Lemme $3, B$ est canoniquement fermé dans $\bar{\Gamma}_{5,2}$, et la topologie induite sur $B$ par la topologie canonique de $\bar{\Gamma}_{5,2}$ est celle de $\overline{\mathbf{R}}^{3}=\mathbf{R}^{3}$.

On définit sur $\bar{\Gamma}_{5,2}$ une topologie des paramètres.

Avec les notations habituelles, on a

$$
\begin{array}{r}
\left(U_{\lambda, \mu, \nu}(F) f \mid g\right) \\
=\int \ldots \int F\left(\rho_{1}, \ldots, \rho_{5}\right) \exp i\left[\nu \frac{\lambda \rho_{3}-\mu \rho_{2}}{\lambda^{2}+\mu^{2}}+\lambda\left(\rho_{4}-\rho_{2} \theta\right)+\mu\left(\rho_{5}-\rho_{3} \theta\right)\right] \\
=\int \ldots \int F\left(\theta+\rho_{1}\right) \overline{g(\theta)} d \rho_{1} \ldots d \rho_{5} d \theta \\
+\mu\left(\rho_{1}-\theta, \rho_{2}, \ldots, \rho_{5}\right) \exp i\left[\nu \frac{\lambda \rho_{3}-\mu \rho_{2}}{\lambda^{2}+\mu^{2}}+\lambda\left(\rho_{4}-\rho_{2} \theta\right)\right] f\left(\rho_{1}\right) \overline{g(\theta)} d \rho_{1} \ldots d \rho_{5} d \theta \\
+
\end{array}
$$

Donc $U_{\lambda, \mu, \nu}(F)$ est défini par le noyau

(6) $K_{\lambda, \mu, \nu}\left(\rho_{1}, \theta\right)$

$$
\begin{aligned}
& =\int \ldots \int F\left(\rho_{1}-\theta, \rho_{2}, \ldots, \rho_{5}\right) \exp i\left[\nu \frac{\lambda \rho_{3}-\mu \rho_{2}}{\lambda^{2}+\mu^{2}}+\lambda\left(\rho_{4}-\rho_{2} \theta\right)\right. \\
& \left.+\mu\left(\rho_{5}-\rho_{3} \theta\right)\right] d \rho_{2} d \rho_{3} d \rho_{4} d \rho_{5} \\
& =\hat{F}_{2345}\left(\rho_{1}-\theta, \frac{\mu \nu}{\lambda^{2}+\mu^{2}}+\lambda \theta,-\frac{\lambda \nu}{\lambda^{2}+\mu^{2}}+\mu \theta,-\lambda,-\mu\right) .
\end{aligned}
$$

Lemme 10. Soit $F \in \mathscr{P}\left(\Gamma_{5,2}\right)$. La fonction $T \rightarrow\|T(F)\|$ est continue sur $\bar{\Gamma}_{5,2}$ pour la topologie des paramètres.

Démonstration. Les raisonnements sont analogues à ceux des Lemmes 5 et 7. Pour pouvoir utiliser le Lemme 4, il faut cette fois observer que

$$
\begin{gathered}
\left(\rho_{1}-\theta\right)^{2}+\left(\frac{\mu \nu}{\lambda^{2}+\mu^{2}}+\lambda \theta\right)^{2}+\left(-\frac{\lambda \nu}{\lambda^{2}+\mu^{2}}+\mu \theta\right)^{2}+\lambda^{2}+\mu^{2} \\
=\left(\rho_{1}-\theta\right)^{2}+\left(\lambda^{2}+\mu^{2}\right) \theta^{2}+\frac{\nu^{2}}{\lambda^{2}+\mu^{2}}+\lambda^{2}+\mu^{2} \\
\geqslant K\left(\rho_{1}{ }^{2}+\theta^{2}\right)
\end{gathered}
$$

pourvu que $\lambda^{2}+\mu^{2}$ soit minoré par une constante $>0$. 
Lemme 11. Soient $A_{1} \subset A, B_{1} \subset B$. On suppose $A_{1} \cup B_{1}$ canoniquement fermé. Alors, si $(0,0,0)$ est adhérent à $A_{1}$ dans $\mathbf{R}^{3}, B_{1}$ contient tout point $(\alpha, \beta, \gamma)$ possédant la propriété suivante: il existe des suites $\beta_{n}, \gamma_{n}, \lambda_{n}, \mu_{n}, \nu_{n}$ de nombres réels tels que

$\beta_{n} \rightarrow \beta, \gamma_{n} \rightarrow \gamma,\left(\lambda_{n}, \mu_{n}, \nu_{n}\right) \rightarrow(0,0,0),\left(\lambda_{n}, \mu_{n}, \gamma_{n}\right) \in A_{1},-\lambda_{n} \gamma_{n}+\mu_{n} \beta_{n}+\gamma_{n}=0$

Démonstration. Soient $\alpha, \beta, \gamma, \beta_{n}, \gamma_{n}, \lambda_{n}, \mu_{n}, \nu_{n}$ avec les propriétés du lemme.

La condition $-\lambda_{n} \gamma_{n}+\mu_{n} \beta_{n}+\nu_{n}=0$ exprime qu'on peut résoudre en $\theta_{n}$ le système:

$$
\lambda_{n} \theta_{n}+\frac{\mu_{n} \nu_{n}}{\lambda_{n}^{2}+\mu_{n}^{2}}=-\beta_{n}, \mu_{n} \theta_{n}-\frac{\lambda_{n} \nu_{n}}{\lambda_{n}^{2}+\mu_{n}^{2}}=-\gamma_{n}
$$

Avec les notations habituelles, on a

$$
\begin{aligned}
\left(U_{\lambda_{n}, \mu_{n}, \nu_{n}}(F) \mathscr{T}\left(-\theta_{n}\right) f \mid\right. & \left.\mathscr{T}\left(-\theta_{n}\right) g\right) \\
& =\int \ldots \int F\left(\rho_{1}, \ldots, \rho_{5}\right) \exp (i \Delta) f\left(\theta+\rho_{1}\right) \overline{g(\theta)} d \rho_{1} \ldots d \rho_{5} d \theta
\end{aligned}
$$

avec

$$
\begin{aligned}
\Delta & =\nu_{n} \frac{\lambda_{n} \rho_{3}-\mu_{n} \rho_{2}}{\lambda_{n}^{2}+\mu_{n}^{2}}+\lambda_{n} \rho_{4}-\lambda_{n} \rho_{2}\left(\theta+\theta_{n}\right)+\mu_{n} \rho_{5}-\mu_{n} \rho_{3}\left(\theta+\theta_{n}\right) \\
& =-\lambda_{n} \rho_{2} \theta-\mu_{n} \rho_{3} \theta+\lambda_{n} \rho_{4}+\mu_{n} \rho_{5}+\beta_{n} \rho_{2}+\gamma_{n} \rho_{3} .
\end{aligned}
$$

Quand $n \rightarrow+\infty$, l'intégrale tend vers

$$
\begin{aligned}
& \int \ldots \int F\left(\rho_{1}, \ldots, \rho_{5}\right) \exp i\left(\beta \rho_{2}+\gamma \rho_{3}\right) f\left(\theta+\rho_{1}\right) \overline{g(\theta)} d \rho_{1} \ldots d \rho_{5} d \theta \\
&=\iint \hat{F}_{2345}\left(\rho_{1},-\beta,-\gamma, 0,0\right) f\left(\theta+\rho_{1}\right) \overline{g(\theta)} d \rho_{1} d \theta=(V f \mid g)
\end{aligned}
$$

où $V$ est, dans $L_{\mathbf{C}}{ }^{2}(\mathbf{R})$, l'opérateur de convolution avec la fonction $\rho_{1} \rightarrow$ $\hat{F}_{2345}\left(-\rho_{1},-\beta,-\gamma, 0,0\right)$. Donc

$$
\mathscr{T}\left(\theta_{n}\right) U_{\lambda_{n}, \mu_{n}, \nu_{n}}(F) \mathscr{T}\left(\theta_{n}\right)^{-1}
$$

tend faiblement vers $V$ quand $n \rightarrow+\infty$, et par suite

$$
\begin{aligned}
\underline{\lim }_{n \rightarrow+\infty}|| U_{\lambda_{n}, \mu_{n}, v_{n}}(F) \| \geqslant \sup _{\xi \in \mathbf{R}}\left|\hat{F}_{12345}(-\xi,-\beta,-\gamma, 0,0)\right| & =\sup _{\xi \in \mathbf{R}}|| V_{\xi, \beta, \gamma}(F) \| .
\end{aligned}
$$

Donc $V_{\alpha, \beta, \gamma} \in B_{1}$.

Proposition 4. Les ensembles canoniquement fermés dans $\bar{\Gamma}_{5,2}$ sont les ensembles $A_{1} \cup B_{1}\left(A_{1} \subset A, B_{1} \subset B\right)$ possédant les propriétés suivantes:

(1) $A_{1}$ est fermé dans $A$ pour la topologie des paramètres;

(2) $B_{1}$ est fermé dans $B$ pour la topologie des paramètres;

(3) Si $(0,0,0)$ est adhérent à $A_{1}$ dans $\mathbf{R}^{3}, B_{1}$ contient tout point $(\alpha, \beta, \gamma)$ tel qu'il existe des suites $\beta_{n}, \gamma_{n}, \lambda_{n}, \mu_{n}, \nu_{n}$, avec $\beta_{n} \rightarrow \beta, \gamma_{n} \rightarrow \gamma,\left(\lambda_{n}, \mu_{n}, \nu_{n}\right) \rightarrow(0,0,0)$, $\left(\lambda_{n}, \mu_{n}, \nu_{n}\right) \in A_{1},-\lambda_{n} \gamma_{n}+\mu_{n} \beta_{n}+\nu_{n}=0$. 
Démonstration. Raisonnant comme pour la Proposition 1, on est ramené à ceci: supposons vérifiées les conditions (1), (2), et (3) de la proposition; soit $T \in \bar{\Gamma}_{5,2}, T \notin A_{1} \cup B_{1}$; et montrons qu'il existe une $F \in \mathscr{S}\left(\Gamma_{5,2}\right)$ telle que $T(F) \neq 0$ et $T^{\prime}(F)=0$ pour $T^{\prime} \in A_{1}$.

(A) Supposons

$$
T=U_{\lambda_{0}, \mu_{0}, \nu_{0}} \notin A_{1} .
$$

Soient $\rho_{1} \in \mathbf{R}, \theta \in \mathbf{R}$. Soit $E$ l'ensemble des points de $\mathbf{R}^{5}$ de la forme

$$
\left(t, \lambda \theta^{\prime}+\frac{\mu \nu}{\lambda^{2}+\mu^{2}}, \mu \theta^{\prime}-\frac{\lambda \nu}{\lambda^{2}+\mu^{2}},-\lambda,-\mu\right),
$$

où

$$
t \in \mathbf{R}, \quad \theta^{\prime} \in \mathbf{R}, \quad(\lambda, \mu, \nu) \in A_{1} .
$$

Comme $A_{1}$ est fermé dans $A$ pour la topologie des paramètres, il est facile de voir que $E$ est fermé dans l'ensemble des points de $\mathbf{R}^{\mathbf{5}}$ dont les deux dernières coordonnées ne sont pas nulles simultanément. Donc

$$
\left(\rho_{1}-\theta, \lambda_{0} \theta+\frac{\mu_{0} \nu_{0}}{\lambda_{0}^{2}+\mu_{0}^{2}}, \mu_{0} \theta-\frac{\lambda_{0} \nu_{0}}{\lambda_{0}^{2}+\mu_{0}^{2}},-\lambda_{0},-\mu_{0}\right)
$$

est extérieur à $E$. D'où l'existence d'une $F \in \mathscr{S}\left(\Gamma_{5,2}\right)$ telle que $\hat{F}_{2345}$ soit non nulle en ce point et nulle sur $E$. Alors,

$$
U_{\lambda_{0}, \mu_{0}, \nu_{0}}(F) \neq 0,
$$

et $U_{\lambda, \mu, \nu}(F)=0$ pour $(\lambda, \mu, \nu) \in A_{1}$.

(B) Supposons $T=V_{\lambda_{0}, \mu_{0}, \nu_{0}} \notin B_{1}$. Alors, $\left(-\lambda_{0},-\mu_{0},-\nu_{0}, 0,0\right)$ n'est pas adhérent à $E$ dans $\mathbf{R}^{5}$. En effet, supposons qu'il existe des suites $t_{n}, \theta_{n}, \lambda_{n}, \mu_{n}$, $\nu_{n}$ avec

$$
\begin{aligned}
t_{n} \rightarrow-\lambda_{0}, \lambda_{n} \theta_{n}+\frac{\mu_{n} \nu_{n}}{\lambda_{n}^{2}+\mu_{n}^{2}} \rightarrow-\mu_{0}, \mu_{n} \theta_{n}-\frac{\lambda_{n} \nu_{n}}{\lambda_{n}^{2}+\mu_{n}^{2}} \rightarrow-\nu_{0}, \\
\lambda_{n} \rightarrow 0, \mu_{n} \rightarrow 0,\left(\lambda_{n}, \mu_{n}, \nu_{n}\right) \in A_{1} .
\end{aligned}
$$

Posons

$$
\beta_{n}=-\lambda_{n} \theta_{n}-\frac{\mu_{n} \nu_{n}}{\lambda_{n}^{2}+\mu_{n}^{2}}, \gamma_{n}=-\mu_{n} \theta_{n}+\frac{\lambda_{n} \nu_{n}}{\lambda_{n}^{2}+\mu_{n}^{2}} .
$$

On a $-\lambda_{n} \gamma_{n}+\mu_{n} \beta_{n}+\nu_{n}=0$, et $\nu_{n}=\lambda_{n} \gamma_{n}-\mu_{n} \beta_{n} \rightarrow 0$. D'après l'hypothèse (3) de la proposition, on a donc $\left(\lambda_{0}, \mu_{0}, \nu_{0}\right) \in B_{1}$, ce qui est contradictoire. Ceci posé, il existe une $F \in \mathscr{S}\left(\Gamma_{5,2}\right)$ telle que $\hat{F}_{12345}$ soit nulle sur $E$ et non nulle en $\left(-\lambda_{0},-\mu_{0},-\nu_{0}, 0,0\right)$. Alors $\hat{F}_{2345}$ est nulle sur $E$, donc $U_{\lambda, \mu, \nu}(F)=0$ pour $(\lambda, \mu, \nu) \in A_{1}$, et

$$
V_{\lambda_{0}, \mu_{0}, \nu_{0}}(F) \neq 0
$$

6. Topologie de $\bar{\Gamma}_{5,3}$. D'après (1, Proposition 7 ), $\bar{\Gamma}_{5,3}$ est réunion de 3 sous-ensembles disjoints $A, B, C$ : 
(1) $A$ est l'ensemble des $U_{\lambda}(\lambda \in \mathbf{R}, \lambda \neq 0)$; chaque $U_{\lambda}$ opère dans $L_{\mathbf{C}}{ }^{2}\left(\mathbf{R}^{2}\right)$ et est définie par la formule

$$
\begin{gathered}
\left(U_{\lambda}(\gamma) f\right)\left(\theta_{1}, \theta_{2}\right)=\exp i \lambda\left(\rho_{5}-\rho_{4} \theta_{1}+\frac{1}{2} \rho_{2} \theta_{1}{ }^{2}-\rho_{3} \theta_{2}\right) f\left(\theta_{1}+\rho_{1}, \theta_{2}+\rho_{2}\right) \\
\left(\gamma=\left(\rho_{1}, \ldots, \rho_{5}\right) \in \Gamma_{5,3}, f \in L_{\mathbf{C}}^{2}\left(\mathbf{R}^{2}\right), \theta_{1}, \theta_{2} \in \mathbf{R}\right) .
\end{gathered}
$$

(2) $B$ est l'ensemble des $V_{\lambda, \mu}(\lambda, \mu \in \mathbf{R}, \lambda \neq 0)$; chaque $V_{\lambda, \mu}$ opère dans $L_{\mathbf{G}}{ }^{2}(\mathbf{R})$ et est définie par la formule

$$
\left(V_{\lambda, \mu}(\gamma) f\right)(\theta)=\exp i\left(\lambda \rho_{4}-\lambda \rho_{2} \theta+\mu \rho_{3}\right) f\left(\theta+\rho_{1}\right) .
$$

(3) $C$ est l'ensemble des $W_{\lambda, \mu, \nu}(\lambda, \mu, \nu \in \mathbf{R})$; chaque $W_{\lambda, \mu, \nu}$ s'identifie à une fonction scalaire sur $\Gamma_{5,3}$, conformément à la relation

$$
W_{\lambda, \mu, \nu}(\gamma)=\exp i\left(\lambda \rho_{1}+\mu \rho_{2}+\nu \rho_{3}\right) .
$$

D'après le Lemme $3, B \cup C$ est canoniquement fermé dans $\bar{\Gamma}_{5,3}$, et la topologie induite sur $B \cup C$ par la topologie canonique de $\bar{\Gamma}_{5,3}$ est la topologie canonique de $\left(\Gamma_{3} \times \mathbf{R}\right)^{-}=\bar{\Gamma}_{3} \times \mathbf{R}$.

On définit sur $\bar{\Gamma}_{5,3}$ une topologie des paramètres.

Avec les notations habituelles, on a

(8) $\left(U_{\lambda}(F) f \mid g\right)=$

$\int \ldots \int F\left(\rho_{1}, \ldots, \rho_{5}\right) \exp i \lambda\left(\rho_{5}-\rho_{4} \theta_{1}+\frac{1}{2} \rho_{2} \theta_{1}^{2}-\rho_{3} \theta_{2}\right) f\left(\theta_{1}+\rho_{1}, \theta_{2}+\rho_{2}\right)$

$$
\overline{g\left(\theta_{1}, \theta_{2}\right)} d \rho_{1} \ldots d \rho_{5} d \theta_{1} d \theta_{2}=
$$

$\int \ldots \int F\left(\rho_{1}-\theta_{1}, \rho_{2}-\theta_{2}, \rho_{3}, \rho_{4}, \rho_{5}\right) \exp (i \lambda A) f\left(\rho_{1}, \rho_{2}\right) \overline{g\left(\theta_{1}, \theta_{2}\right)} d \rho_{1} \ldots d \rho_{5} d \theta_{1} d \theta_{2}$

où

$$
A=\rho_{5}-\rho_{4} \theta_{1}+\frac{1}{2} \rho_{2} \theta_{1}^{2}-\frac{1}{2} \theta_{2} \theta_{1}^{2}-\rho_{3} \theta_{2} .
$$

Donc $U_{\lambda}(F)$ est défini par le noyau

(9) $K_{\lambda}\left(\rho_{1}, \rho_{2}, \theta_{1}, \theta_{2}\right)$

$$
\begin{aligned}
& =\iiint F\left(\rho_{1}-\theta_{1}, \rho_{2}-\theta_{2}, \rho_{3}, \rho_{4}, \rho_{5}\right) \exp (i \lambda A) d \rho_{3} d \rho_{4} d \rho_{5} \\
& =\exp \frac{1}{2} i \lambda\left(\rho_{2}-\theta_{2}\right) \theta_{1}^{2} . \hat{F}_{345}\left(\rho_{1}-\theta_{1}, \rho_{2}-\theta_{2}, \lambda \theta_{2}, \lambda \theta_{1},-\lambda\right) .
\end{aligned}
$$

Lemme 12. Soit $F \in \mathscr{S}\left(\Gamma_{5,3}\right)$. La fonction $T \rightarrow\|T(F)\|$ est continue sur $\Gamma_{5.3}$ pour la topologie des paramètres.

Démonstration. D'après le Lemme 4 , la fonction $\left(\rho_{1}, \rho_{2}, \theta_{1}, \theta_{2}\right) \rightarrow \hat{F}_{345}\left(\rho_{1}-\theta_{1}\right.$, $\left.\rho_{2}-\theta_{2}, \lambda \theta_{2}, \lambda \theta_{1},-\lambda\right)$ est un élément de $\left.L_{\mathbf{C}^{2}}{ }^{2} \mathbf{R}^{4}\right)$ qui dépend continûment de $\lambda \in \mathbf{R}-\{0\}$ pour la topologie forte. D'autre part, l'opérateur unitaire de multiplication par $\exp \frac{1}{2} i \lambda\left(\rho_{2}-\theta_{2}\right) \theta_{1}{ }^{2}$ dans $L_{\mathbf{C}}{ }^{2}\left(\mathbf{R}^{4}\right)$ dépend continûment de $\lambda$ pour la topologie forte. Donc l'application $\lambda \rightarrow K_{\lambda}$ de $\mathbf{R}-\{0\}$ dans $L_{\mathbf{C}}{ }^{2}\left(\mathbf{R}^{4}\right)$ est fortement continue. On achève alors comme pour le Lemme 5 . 
Lemme 13. Soient $A_{1} \subset A, B_{1} \subset B, C_{1} \subset C$. On suppose $A_{1} \cup B_{1} \cup C_{1}$ canoniquement fermé. Si 0 est adhérent à $A_{1}$ dans $\mathbf{R}$, on a $B_{1}=B, C_{1}=C$.

Démonstration. Soient $\beta_{1}, \beta_{2} \in \mathbf{R}$. Avec les notations habituelles, on a

$$
\begin{gathered}
\left(U_{\lambda}(F) \mathscr{T}\left(-\frac{\beta_{1}}{\lambda},-\frac{\beta_{2}}{\lambda}\right) \mathscr{M}\left(\exp \left(-\frac{1}{2} i \frac{\beta_{1}^{2}}{\lambda} \theta_{2}\right)\right) f \mid \mathscr{T}\left(-\frac{\beta_{1}}{\lambda},-\frac{\beta_{2}}{\lambda}\right)\right. \\
\left.\mathscr{M}\left(\exp \left(-\frac{1}{2} i \frac{\beta_{1}^{2}}{\lambda} \theta_{2}\right)\right) g\right) \\
=\int \ldots \int F\left(\rho_{1}, \ldots, \rho_{5}\right) \exp i \lambda\left(\rho_{5}-\rho_{4} \theta_{1}+\frac{1}{2} \rho_{2} \theta_{1}^{2}-\rho_{3} \theta_{2}\right) \\
\exp -\frac{1}{2} i \frac{\beta_{1}^{2}}{\lambda}\left(\theta_{2}-\frac{\beta_{2}}{\lambda}+\rho_{2}\right) \\
f\left(\theta_{1}-\frac{\beta_{1}}{\lambda}+\rho_{1}, \theta_{2}-\frac{\beta_{2}}{\lambda}+\rho_{2}\right) \exp \frac{1}{2} i \frac{\beta_{1}^{2}}{\lambda}\left(\theta_{2}-\frac{\beta_{2}}{\lambda}\right) g\left(\theta_{1}-\frac{\beta_{1}}{\lambda}, \theta_{2}-\frac{\beta_{2}}{\lambda}\right) \\
=\int \ldots \int F\left(\rho_{1}, \ldots, \rho_{5}\right) \exp (i \Delta) f\left(\theta_{1}+\rho_{1}, \theta_{2}+\rho_{2}\right) \overline{g\left(\theta_{1}, \theta_{2}\right)} d \rho_{1} \ldots d \theta_{2} \\
\int \ldots \rho_{5} d \theta_{1} d \theta_{2}
\end{gathered}
$$

avec

$$
\begin{aligned}
\Delta & =\lambda \rho_{5}-\lambda \rho_{4}\left(\theta_{1}+\frac{\beta_{1}}{\lambda}\right)+\frac{1}{2} \lambda \rho_{2}\left(\theta_{1}+\frac{\beta_{1}}{\lambda}\right)^{2}-\lambda \rho_{3}\left(\theta_{2}+\frac{\beta_{2}}{\lambda}\right)-\frac{1}{2} \frac{\beta_{1}{ }^{2}}{\lambda} \rho_{2} \\
& =\lambda \rho_{5}-\lambda \rho_{4} \theta_{1}+\frac{1}{2} \lambda \rho_{2} \theta_{1}{ }^{2}-\lambda \rho_{3} \theta_{2}-\beta_{1} \rho_{4}+\beta_{1} \rho_{2} \theta_{1}-\beta_{2} \rho_{3} .
\end{aligned}
$$

Quand $\lambda \rightarrow 0$, l'intégrale tend vers

$$
\begin{aligned}
\int \ldots \int F\left(\rho_{1}, \ldots, \rho_{5}\right) \exp i\left(-\beta_{2} \rho_{3}-\beta_{1} \rho_{4}+\beta_{1} \rho_{2} \theta_{1}\right) f\left(\theta_{1}+\rho_{1}, \theta_{2}+\rho_{2}\right) \\
\overline{g\left(\theta_{1}, \theta_{2}\right)} d \rho_{1} \ldots d \rho_{5} d \theta_{1} d \theta_{2} .
\end{aligned}
$$

D'autre part, on peut identifier canoniquement $L_{\mathbf{G}}{ }^{2}\left(\mathbf{R}^{2}\right)$ à l'espace des fonctions de carré intégrable sur $\mathbf{R}$ pour la mesure de Lebesgue et à valeurs dans $L_{\mathbf{C}}{ }^{2}(\mathbf{R})$ c'est-à-dire encore à l'intégrale hilbertienne

$$
\int_{\mathbf{R}}^{\oplus} \mathfrak{S}_{t} d t
$$

où, pour tout $t, \mathfrak{S}_{t}=L_{\mathbf{C}}{ }^{2}(\mathbf{R})$; dans cette identification, l'élément $\left(\theta_{1}, \theta_{2}\right) \rightarrow$ $f\left(\theta_{1}, \theta_{2}\right)$ de $L_{\mathbf{C}^{2}}\left(\mathbf{R}^{2}\right)$ correspond au champ de vecteurs $\theta_{2} \rightarrow f_{\theta_{2}}$, où $f_{\theta_{2}}$ est l'élément de $L_{\mathbf{C}}{ }^{2}(\mathbf{R})$ défini (pour presque tout $\theta_{2}$ ) par

$$
f_{\theta_{2}}\left(\theta_{1}\right)=f\left(\theta_{1}, \theta_{2}\right) \text {. }
$$


Supposons $\beta_{1} \neq 0$. Pour chaque $\theta_{2} \in \mathbf{R}$, considérons l'opérateur

$$
\mathscr{T}\left(-\frac{\theta_{2}}{\beta_{1}}\right)_{-\beta_{1},-\beta_{2}}(F) \mathscr{T}\left(\frac{\theta_{2}}{\beta_{1}}\right)
$$

dans

$$
\mathfrak{S}_{\theta_{2}}=L_{\mathbf{C}}^{2}(\mathbf{R}) \text {. }
$$

Cet opérateur a une norme indépendante de $\theta_{2}$ et dépend continûment $\theta_{2}$ pour la topologie forte. L'application

$$
\theta_{2} \rightarrow \mathscr{T}\left(-\frac{\theta_{2}}{\beta_{1}}\right) V_{-\beta_{1},-\beta_{2}}(F) \mathscr{T}\left(\frac{\theta_{2}}{\beta_{1}}\right)
$$

est donc un champ continu borné d'opérateurs, et définit dans

$$
\int_{\mathbf{R}}^{\oplus} \mathfrak{W}_{\theta_{2}} d \theta_{2}=L_{\mathbf{C}}^{2}\left(\mathbf{R}^{2}\right)
$$

un opérateur $S$, de norme

$$
\left\|V_{-\beta_{1},-\beta_{\mathbf{2}}}(F)\right\|
$$

tel que

$$
\begin{gathered}
(S f \mid g)=\int\left(V_{-\beta_{1},-\beta_{2}}(F) \mathscr{T}\left(\frac{\theta_{2}}{\beta_{1}}\right) f_{\theta_{2}} \mathscr{T} \mid\left(\frac{\theta_{2}}{\beta_{1}}\right) g_{\theta_{2}}\right) d \theta_{2} \\
=\int \ldots \int F\left(\rho_{1}, \ldots, \rho_{5}\right) \exp i\left(-\beta_{2} \rho_{3}-\beta_{1} \rho_{4}+\beta_{1} \rho_{2} \theta_{1}\right) f\left(\theta_{1}+\rho_{1}+\frac{\theta_{2}}{\beta_{1}}, \theta_{2}\right) \\
\frac{g\left(\theta_{1}+\frac{\theta_{2}}{\beta_{1}}, \theta_{2}\right)}{2} d \rho_{1} \ldots d \rho_{5} d \theta_{1} d \theta_{2} \\
=\int \ldots \int F\left(\rho_{1}, \ldots, \rho_{5}\right) \exp i\left(-\beta_{2} \rho_{3}-\beta_{1} \rho_{4}+\beta_{1} \rho_{2} \theta_{1}\right) \exp \left(-i \rho_{2} \theta_{2}\right) \\
f\left(\theta_{1}+\rho_{1}, \theta_{2}\right) \overline{g\left(\theta_{1}, \theta_{2}\right)} d \rho_{1} \ldots d \rho_{5} d \theta_{1} d \theta_{2} .
\end{gathered}
$$

Désignons par $\mathscr{F}_{2}$ la transformation de Fourier par rapport à la 2e variable dans $L_{\mathbf{G}}{ }^{2}\left(\mathbf{R}^{2}\right)$. D'après la formule de Plancherel, on a

$$
\begin{aligned}
\int \exp \left(-i \rho_{2} \theta_{2}\right) f\left(\theta_{1}+\rho_{1}, \theta_{2}\right) \overline{g\left(\theta_{1}, \theta_{2}\right)} d \theta_{2} \\
\quad=\frac{1}{2 \pi} \int\left(\mathscr{F}_{2} f\right)\left(\theta_{1}+\rho_{1}, \theta_{2}+\rho_{2}\right) \overline{\left(\mathscr{F}_{2} g\right)\left(\theta_{1}, \theta_{2}\right)} d \theta_{2} .
\end{aligned}
$$

Donc

$$
\begin{aligned}
& \left(\mathscr{F}_{2} S \mathscr{F}_{2}^{-1} f \mid g\right)=2 \pi\left(S \mathscr{F}_{2}^{-1} f \mid \mathscr{F}_{2}^{-1} g\right) \\
& \quad=\int \ldots \int F\left(\rho_{1}, \ldots, \rho_{5}\right) \exp i\left(-\beta_{2} \rho_{3}-\beta_{1} \rho_{4}+\frac{\left.\beta_{1} \rho_{2} \theta_{1}\right) f\left(\theta_{1}+\rho_{1}, \theta_{2}+\rho_{2}\right)}{\overline{g\left(\theta_{1}, \theta_{2}\right)} d \rho_{1} \ldots d \rho_{5} d \theta_{1} d \theta_{2} .}\right.
\end{aligned}
$$


En définitive, quand $\lambda \rightarrow 0$, l'opérateur

$$
\mathscr{M}\left(\exp \left(\frac{1}{2} i \frac{\beta_{1}{ }^{2}}{\lambda} \theta_{2}\right)\right) \mathscr{T}\left(\frac{\beta_{1}}{\lambda}, \frac{\beta_{2}}{\lambda}\right) U_{\lambda}(F) \mathscr{T}\left(\frac{\beta_{1}}{\lambda}, \frac{\beta_{2}}{\lambda}\right)^{-1} \mathscr{M}\left(\exp \left(\frac{1}{2} i \frac{\beta_{1}{ }^{2}}{\lambda} \theta_{2}\right)\right)^{-1}
$$

tend faiblement vers $\mathscr{F}_{2} S \mathscr{F}_{2}{ }^{-1}$. Par suite,

$$
\underline{\lim }_{\lambda \rightarrow 0}\left\|U_{\lambda}(F)\right\| \geqslant\|S\|=\left\|V_{-\beta_{1},-\beta_{\mathbf{2}}}(F)\right\| \text {. }
$$

Donc $V_{\lambda, \mu} \in B_{1}$ quels que soient $\lambda$ et $\mu$, ce qui prouve que $B_{1}=B$. Comme $B_{1}$ est partout dense dans $B \cup C$ pour la topologie canonique d'après la Proposition 1, on a aussi $C_{1}=C$.

Proposition 5. Les ensembles canoniquement fermés dans $\bar{\Gamma}_{5,3}$ sont les ensembles $A_{1} \cup B_{1} \cup C_{1}\left(A_{1} \subset A, B_{1} \subset B, C_{1} \subset C\right)$ possédant les propriétés suivantes:

(1) $A_{1}$ est fermé dans $A$ pour la topologie des paramètres;

(2) $B_{1} \cup C_{1}$ est canoniquement fermé dans $B \cup C=\bar{\Gamma}_{3} \times \mathbf{R}$;

(3) Si 0 est adhérent à $A_{1}$ dans $\mathbf{R}$, on a $B_{1}=B, C_{1}=C$.

Démonstration. Supposons remplies les conditions (1), (2), et (3) de la proposition. Soit $T \in \bar{\Gamma}_{5,3}$ avec $T \notin A_{1} \cup B_{1} \cup C_{1}$. Il s'agit de construire une $F \in \mathscr{S}\left(\Gamma_{5,3}\right)$ telle que $T(F) \neq 0$ et $T^{\prime}(F)=0$ pour $T^{\prime} \in A_{1}$.

(A) Supposons $T=U_{\lambda_{0}} \notin A_{1}$. Il existe $F \in \mathscr{S}\left(\Gamma_{5,3}\right)$ telle que $\hat{F}_{345}$ soit nulle lorsque la $5 \mathrm{e}$ variable appartient à $A_{1}$ et non nulle lorsque la $5 \mathrm{e}$ variable a pour valeur $\lambda_{0}$. Alors, compte tenu de (9), on a $U_{\lambda_{0}}(F) \neq 0$ et $U_{\lambda}(F)=0$ pour $\lambda \in A_{1}$.

(B) Supposons $T=V_{\lambda_{0}, \mu_{0}} \notin B_{1}$. D'après la condition (3) de la proposition, 0 est non adhérent à $A_{1}$ dans $\mathbf{R}$. Donc il existe $F \in \mathscr{P}\left(\Gamma_{5,3}\right)$ telle que $\hat{F}_{2345}\left(\rho_{1}-\theta, \lambda_{0} \theta,-\mu_{0},-\lambda_{0}, 0\right) \neq 0$ pour certaines valeurs de $\rho_{1}$ et $\theta$, et telle que $\hat{F}_{2345}$ (donc aussi $\hat{F}_{345}$ ) soit nulle lorsque la 5 e variable appartient à $A_{1}$. La première condition entraîne facilement que $V_{\lambda_{0}, \mu_{0}}(F) \neq 0$; la deuxième entraîne que $U_{\lambda}(F)=0$ pour $\lambda \in A_{1}$.

(C) Si $T=W_{\lambda_{0}, \mu_{0}, \nu_{0}} \in C_{1}$, le raisonnement est analogue à celui de (B).

7. Topologie de $\bar{\Gamma}_{5,5}$. D'après (1, Proposition 9 ), $\bar{\Gamma}_{5,5}$ est réunion de 3 sous-ensembles disjoints $A, B, C$;

(1) $A$ est l'ensemble des $U_{\lambda, \mu, \nu, \rho}\left(\lambda, \mu, \nu, \rho \in \mathbf{R}, \mu^{3}-\nu^{2}+\lambda^{2} \rho=0\right.$, $\left.\lambda^{2}+\mu^{2}+\nu^{2} \neq 0\right)$; chaque $U_{\lambda, \mu, \nu, \rho}$ opère dans $L_{\mathbf{C}}{ }^{2}(\mathbf{R})$ et est définie par la formule

$$
\begin{aligned}
& \left(U_{\lambda, \mu, \nu, \rho}(\gamma) f\right)(\theta)= \\
& \quad \exp i\left(-\frac{1}{3} \frac{\nu}{\lambda^{2}} \rho_{2}-\frac{1}{2} \frac{\mu}{\lambda}\left(\rho_{3}-\rho_{2} \theta\right)+\lambda\left(\rho_{5}-\rho_{4} \theta+\frac{1}{2} \rho_{3} \theta^{2}-\frac{1}{6} \rho_{2} \theta^{3}\right)\right) f\left(\theta+\rho_{1}\right)
\end{aligned}
$$

si $\lambda \neq 0$, et par la formule

$$
\left(U_{\lambda, \mu, \nu, \rho}(\gamma) f\right)(\theta)=\exp i\left(-\frac{1}{6} \frac{\rho}{\nu} \rho_{2}-\frac{\nu}{\mu}\left(\rho_{4}-\rho_{3} \theta+\frac{1}{2} \rho_{2} \theta^{2}\right)\right) f\left(\theta+\rho_{1}\right)
$$

si $\lambda=0$. 


$$
\left(\gamma=\left(\rho_{1}, \ldots, \rho_{5}\right) \in \Gamma_{5,5}, f \in L_{\mathbf{C}}^{2}(\mathbf{R}), \theta \in \mathbf{R}\right) .
$$

(2) $B$ est l'ensemble des $V_{\lambda}(\lambda \in \mathbf{R}, \lambda \neq 0)$; chaque $V_{\lambda}$ opère dans $L_{\mathbf{C}}{ }^{2}(\mathbf{R})$ et est définie par la formule

$$
\left(V_{\lambda}(\gamma) f\right)(\theta)=\exp i \lambda\left(\rho_{3}-\rho_{2} \theta\right) f\left(\theta+\rho_{1}\right) .
$$

(3) $C$ est l'ensemble des $W_{\lambda, \mu}(\lambda, \mu \in \mathbf{R})$; chaque $W_{\lambda, \mu}$ s'identifie à une fonction scalaire sur $\Gamma_{5,5}$ conformément à la relation

$$
W_{\lambda, \mu}(\gamma)=\exp i\left(\lambda \rho_{1}+\mu \rho_{2}\right) .
$$

D'après le Lemme $3, B \cup C$ est canoniquement fermé dans $\bar{\Gamma}_{5,5}$, et la topologie induite sur $B \cup C$ par la topologie canonique de $\bar{\Gamma}_{5,5}$ est la topologie de $\bar{\Gamma}_{3}$.

On définit sur $\bar{\Gamma}_{5,5}$ une topologie des paramètres.

Avec les notations habituelles, on a, pour $\lambda \neq 0$

(10) $\left(U_{\lambda, \mu, \nu, \rho}(F) f \mid g\right)$

$$
\begin{aligned}
& =\int \ldots \int F\left(\rho_{1}, \ldots, \rho_{5}\right) \exp (i A) f\left(\theta+\rho_{1}\right) \overline{g(\theta)} d \rho_{1} \ldots d \rho_{5} d \theta \\
& =\int \ldots \int F\left(\rho_{1}-\theta, \rho_{2}, \ldots, \rho_{5}\right) \exp (i A) f\left(\rho_{1}\right) \overline{g(\theta)} d \rho_{1} \ldots d \rho_{5} d \theta
\end{aligned}
$$

avec

$$
A=-\frac{1}{3} \frac{\nu}{\lambda^{2}} \rho_{2}-\frac{1}{2} \frac{\mu}{\lambda}\left(\rho_{3}-\rho_{2} \theta\right)+\lambda\left(\rho_{5}-\rho_{4} \theta+\frac{1}{2} \rho_{3} \theta^{2}-\frac{1}{6} \cdot \rho_{2} \theta^{3}\right) .
$$

Donc $U_{\lambda, \mu, \nu, \rho}(F)$ est défini, pour $\lambda \neq 0$, par le noyau

$$
\begin{aligned}
\int \ldots \int F\left(\rho_{1}-\theta, \rho_{2}, \ldots, \rho_{5}\right) \exp (i A) d \rho_{2} d \rho_{3} d \rho_{4} d \rho_{5} \\
=\hat{F}_{2345}\left(\rho_{1}-\theta, \frac{1}{3} \frac{\nu}{\lambda^{2}}-\frac{1}{2} \frac{\mu}{\lambda} \theta+\frac{1}{6} \lambda \theta^{3}, \frac{1}{2} \frac{\mu}{\lambda}-\frac{1}{2} \lambda \theta^{2}, \lambda \theta,-\lambda\right) .
\end{aligned}
$$

Pour $\lambda=0$, on a

$$
\begin{aligned}
& \left(U_{\lambda, \mu, \nu, \rho}(F) f \mid g\right) \\
& =\int \ldots \int F\left(\rho_{1}, \ldots, \rho_{5}\right) \exp i\left(-\frac{1}{6} \frac{\rho}{\nu} \rho_{2}-\frac{\nu}{\mu}\left(\rho_{4}-\rho_{3} \theta+\frac{1}{2} \rho_{2} \theta^{2}\right)\right) \\
& f\left(\theta+\rho_{1}\right) \overline{g(\theta)} d \rho_{1} \ldots d \rho_{5} d \theta \\
& =\int \ldots \int F\left(\rho_{1}-\theta, \rho_{2}, \ldots, \rho_{5}\right) \exp i\left(-\frac{1}{6} \frac{\rho}{\nu} \rho_{2}-\frac{\nu}{\mu}\left(\rho_{4}-\rho_{3} \theta+\frac{1}{2} \rho_{2} \theta^{2}\right)\right) \\
& f\left(\rho_{1}\right) \overline{g(\theta)} d \rho_{1} \ldots d \rho_{5} d \theta .
\end{aligned}
$$


Donc $U_{\lambda, \mu, \nu, \rho}(F)$ est défini, pour $\lambda=0$, par le noyau

$$
\begin{gathered}
\int \ldots \int F\left(\rho_{1}-\theta, \rho_{2}, \ldots, \rho_{5}\right) \exp i\left(-\frac{1}{6} \frac{\rho}{\nu} \rho_{2}-\frac{\nu}{\mu}\left(\rho_{4}-\rho_{3} \theta+\frac{1}{2} \rho_{2} \theta^{2}\right)\right) \\
\quad \hat{F}_{2345}\left(\rho_{2} \ldots d \rho_{5}-\theta, \frac{1}{6} \frac{\rho}{\nu}+\frac{1}{2} \frac{\nu}{\mu} \theta^{2},-\frac{\nu}{\mu} \theta, \frac{\nu}{\mu}, 0\right) .
\end{gathered}
$$

Nous nous contenterons de démontrer le résultat suivant:

Proposition 6. La topologie induite sur $A$ par la topologie canonique est la topologie des paramètres.

Démonstration. Soit $A_{1}$ une partie de $A$ canoniquement fermée dans $A$, et montrons que $A_{1}$ est fermée dans $A$ pour la topologie des paramètres. Soit $\left(\lambda_{n}, \mu_{n}, \nu_{n}, \rho_{n}\right)$ une suite de points de $A_{1}$ telle que $\lambda_{n}, \mu_{n}, \nu_{n}, \rho_{n}$ aient des limites finies $\lambda, \mu, \nu, \rho$, où $\lambda, \mu, \nu$ ne sont pas simultanément nuls. Il s'agit de montrer que $(\lambda, \mu, \nu, \rho) \in A_{1}$.

Si $\lambda \neq 0$, la formule (10) montre que

$$
\left(U_{\lambda_{n}, \mu_{n}, \nu_{n}, \rho_{n}}(F) f \mid g\right) \rightarrow\left(U_{\lambda, \mu, \nu, \rho}(F) f \mid g\right),
$$

donc que

$$
U_{\lambda_{n}, \mu_{n}, \nu_{n}, \rho_{n}}(F)
$$

tend faiblement vers $U_{\lambda, \mu, \nu, \rho}(F)$, donc que

$$
\underline{\lim }_{n \rightarrow+\infty}\left\|U_{\lambda_{n}, \mu_{n}, \nu_{n}, \rho_{n}}(F) \geqslant\right\| U_{\lambda, \mu, \nu, \rho}(F) \| .
$$

Alors $U_{\lambda, \mu, \nu, \rho}$ est canoniquement adhérent à $A_{1}$ (Lemme 1), donc $U_{\lambda, \mu, \nu, \rho} \in A_{1}$. On raisonne de même si $\lambda=0$ et si $\lambda_{n}=0$ pour une infinité de valeurs de $n$.

Supposons maintenant $\lambda=0$, et $\lambda_{n} \neq 0$ pour une infinité de valeurs de $n$, donc, en changeant de notations, pour tout $n$. On a $\mu^{3}-\nu^{2}=0$, donc $\mu>0$. Faisons d'abord l'hypothèse que $\nu<0$, d'où $\mu(\mu)^{\frac{1}{2}}=-\nu$. On a $\mu_{n}>0$ pour $n$ assez grand, et

$$
\begin{aligned}
\left(U_{\lambda_{n}, \mu_{n}, \nu_{n}, \rho_{n}}(F) \mathscr{T}\left(\frac{\mu_{n}^{\frac{1}{2}}}{\lambda_{n}}\right) f \mid \mathscr{T}\left(\frac{\mu_{n}^{\frac{1}{2}}}{\lambda_{n}}\right) g\right) & \\
& =\int \ldots \int F\left(\rho_{1}, \ldots, \rho_{5}\right) \exp (i \Delta) f\left(\theta+\rho_{1}\right) \overline{g(\theta)} d \rho_{1} \ldots d \rho_{5} d \theta
\end{aligned}
$$

avec

$$
\begin{aligned}
& \Delta=-\frac{1}{3} \frac{\nu_{n}}{\lambda_{n}{ }^{2}} \rho_{2}-\frac{1}{2} \frac{\mu_{n}}{\lambda_{n}} \rho_{3}+\frac{1}{2} \frac{\mu_{n}}{\lambda_{n}} \rho_{2}\left(\theta-\frac{\mu_{n}^{\frac{1}{2}}}{\lambda_{n}}\right)+\lambda_{n} \rho_{5}-\lambda_{n} \rho_{4}\left(\theta-\frac{\mu_{n}^{\frac{1}{2}}}{\lambda_{n}}\right) \\
& +\frac{1}{2} \lambda_{n} \rho_{3}\left(\theta-\frac{\mu_{n}^{\frac{1}{2}}}{\lambda_{n}}\right)^{2}-\frac{1}{6} \lambda_{n} \rho_{2}\left(\theta-\frac{\mu_{n}^{\frac{1}{2}}}{\lambda_{n}}\right)^{3} \\
& =-\frac{1}{3} \frac{\nu_{n}+\mu_{n} \mu_{n}^{\frac{1}{2}}}{\lambda_{n}^{2}} \rho_{2}+\lambda_{n}\left(\rho_{5}-\rho_{4} \theta+\frac{1}{2} \rho_{3} \theta^{2}-\frac{1}{6} \rho_{2} \theta^{3}\right)+\mu_{n}^{\frac{1}{2}}\left(\rho_{4}-\rho_{3} \theta+\frac{1}{2} \rho_{2} \theta^{2}\right) \\
& =-\frac{1}{3} \frac{\rho_{n}}{\nu_{n}-\mu_{n} \mu_{n}^{\frac{3}{3}}} \rho_{2}+\lambda_{n}\left(\rho_{5}-\rho_{4} \theta+\frac{1}{2} \rho_{3} \theta^{2}-\frac{1}{6} \rho_{2} \theta^{3}\right)+\mu_{n}^{\frac{1}{2}}\left(\rho_{4}-\rho_{3} \theta+\frac{1}{2} \rho_{2} \theta^{2}\right) \text {. }
\end{aligned}
$$


Donc, quand $n \rightarrow+\infty$,

$$
\begin{aligned}
\left(U_{\lambda_{n}, \mu_{n}, \nu_{n}, \rho_{n}}(F)\right. & \left.\mathscr{T}\left(\frac{\mu_{n}^{\frac{1}{2}}}{\lambda_{n}}\right) f \mid \mathscr{T}\left(\frac{\underline{\mu}_{n}^{\frac{1}{2}}}{\lambda_{n}}\right) g\right) \rightarrow \\
& \int \ldots \int F\left(\rho_{1}, \ldots, \rho_{5}\right) \exp i\left(-\frac{1}{6} \frac{\rho}{\nu} \rho_{2}-\frac{\nu}{\mu}\left(\rho_{4}-\rho_{3} \theta+\frac{1}{2} \rho_{2} \theta^{2}\right)\right) \\
& f\left(\theta+\rho_{1}\right) \overline{g(\theta)} d \rho_{1} \ldots d \rho_{5} d \theta=\left(U_{\lambda, \mu, \nu, \rho}(F) f \mid g\right),
\end{aligned}
$$

et le raisonnement s'achève comme plus haut. Enfin, si $\nu>0$, il suffit de changer partout $\mu_{n}^{\frac{1}{2}}$ en $-\mu_{n}^{\frac{1}{2}}$ dans ce qui précède.

Pour établir que, réciproquement, une partie de $A$ fermée pour la topologie des paramètres est canoniquement fermée dans $A$, nous aurons besoin du lemme suivant.

Lemme 14. Soit $A_{1}$ une partie de A fermée pour la topologie des paramètres. Soit $E_{1}$ l'ensemble des points de $\mathbf{R}^{4}$ de la forme

$$
\left(\frac{1}{3} \frac{\nu}{\lambda^{2}}-\frac{1}{2} \frac{\mu}{\lambda} \theta+\frac{1}{6} \lambda \theta^{3}, \quad \frac{1}{2} \frac{\mu}{\lambda}-\frac{1}{2} \lambda \theta^{2}, \quad \lambda \theta,-\lambda\right)
$$

oì $\theta \in \mathbf{R},(\lambda, \mu, \nu, \rho) \in A_{1}, \lambda \neq 0$. Soit $E_{2}$ l'ensemble des points de $\mathbf{R}^{4}$ de la forme

$$
\left(\frac{1}{6} \frac{\rho}{\nu}+\frac{1}{2} \frac{\nu}{\mu} \theta^{2}, \quad-\frac{\nu}{\mu} \theta, \quad \frac{\nu}{\mu}, 0\right)
$$

où $\theta \in \mathbf{R},(0, \mu, \nu, \rho) \in A_{1}$. Alors, $E_{1} \cup E_{2}$ est fermé dans $\mathbf{R}^{4}-\Omega$, en désignant par $\Omega$ l'ensemble des points de $\mathbf{R}^{4}$ dont les 2 dernières coordonnées sont nulles.

Démonstration. (A) Supposons d'abord que

$$
\frac{1}{3} \frac{\nu_{n}}{\lambda_{n}^{2}}-\frac{1}{2} \frac{\mu_{n}}{\lambda_{n}} \theta_{n}+\frac{1}{6} \lambda_{n} \theta_{n}^{3} \rightarrow \xi_{1}, \frac{1}{2} \frac{\mu_{n}}{\lambda_{n}}-\frac{1}{2} \lambda_{n} \theta_{n}^{2} \rightarrow \xi_{2}, \lambda_{n} \theta_{n} \rightarrow \xi_{3},-\lambda_{n} \rightarrow \xi_{4},
$$

où $\left(\lambda_{n}, \mu_{n}, \nu_{n}, \rho_{n}\right) \in A_{1}, \lambda_{n} \neq 0$, et où $\xi_{1}, \xi_{2}, \xi_{3}, \xi_{4}$ sont des nombres réels finis tels que $\xi_{3}$ et $\xi_{4}$ ne soient pas nuls tous les deux. Montrons que $\left(\xi_{1}, \xi_{2}, \xi_{3}, \xi_{4}\right)$ $\in E_{1} \cup E_{2}$.

Si $\xi_{4} \neq 0, \theta_{n}$, donc, $\mu_{n} / \lambda_{n}$ donc $\nu_{n} / \lambda_{n}^{2}$, donc $\mu_{n}$ et $\nu_{n}$, ont des limites finies, donc

$$
\rho_{n}=\frac{\nu_{n}^{2}-\mu_{n}^{3}}{\lambda_{n}^{2}}
$$

a une limite finie. Soient $\lambda, \mu, \nu, \rho, \theta$ les limites de $\lambda_{n}, \mu_{n}, \nu_{n}, \rho_{n}, \theta_{n}$. On a $(\lambda, \mu, \nu, \rho) \in A_{1}$, et

$$
\frac{1}{3} \frac{\nu}{\lambda^{2}}-\frac{1}{2} \frac{\mu}{\lambda} \theta+\frac{1}{6} \lambda \theta^{3}=\xi_{1}, \frac{1}{2} \frac{\mu}{\lambda}-\frac{1}{2} \lambda \theta^{2}=\xi_{2}, \lambda \theta=\xi_{3},-\lambda=\xi_{4},
$$

donc $\left(\xi_{1}, \xi_{2}, \xi_{3}, \xi_{4}\right) \in E_{1}$. 
Si $\xi_{4}=0$ (donc $\xi_{3} \neq 0$ ), on a $\mu_{n}-\left(\lambda_{n} \theta_{n}\right)^{2} \rightarrow 0$, donc $\mu_{n} \rightarrow \xi_{3}{ }^{2}>0$, et $2 \nu_{n}-3 \mu_{n} \lambda_{n} \theta_{n}+\left(\lambda_{n} \theta_{n}\right)^{3} \rightarrow 0$, donc $\nu_{n} \rightarrow \xi_{3}^{3}$. Par ailleurs,

$$
\begin{aligned}
& 9\left(\frac{1}{3} \frac{\nu_{n}}{\lambda_{n}^{2}}-\frac{1}{2} \frac{\mu_{n}}{\lambda_{n}} \theta_{n}+\frac{1}{6} \lambda_{n} \theta_{n}^{3}\right)^{2} \lambda_{n}^{2}-8\left(\frac{1}{2} \frac{\mu_{n}}{\lambda_{n}}-\frac{1}{2} \lambda_{n} \theta_{n}^{2}\right)^{3} \lambda_{n} \rightarrow 0 \\
& 18\left(\frac{1}{3} \frac{\nu_{n}}{\lambda_{n}^{2}}-\frac{1}{2} \frac{\mu_{n}}{\lambda_{n}} \theta_{n}+\frac{1}{6} \lambda_{n} \theta_{n}^{3}\right)\left(\frac{1}{2} \frac{\mu_{n}}{\lambda_{n}}-\frac{1}{2} \lambda_{n} \theta_{n}^{2}\right) \lambda_{n}^{2} \theta_{n} \rightarrow 0 \\
& 3 \lambda_{r}^{2} \theta_{n}^{2}\left[2\left(\frac{1}{3} \frac{\nu_{n}}{\lambda_{n}^{2}}-\frac{1}{2} \frac{\mu_{n}}{\lambda_{n}} \theta_{n}+\frac{1}{6} \lambda_{n} \theta_{n}^{3}\right) \lambda_{n} \theta_{n}-\left(\frac{1}{2} \frac{\mu_{n}}{\lambda_{n}}-\frac{1}{2} \lambda_{n} \theta_{n}^{2}\right)^{2}\right] \\
& \rightarrow 3 \xi_{3}^{2}\left(2 \xi_{1} \xi_{3}-\xi_{2}^{2}\right) .
\end{aligned}
$$

Additionnant, on trouve que

$$
\frac{\nu_{n}^{2}-\mu_{n}^{3}}{\lambda_{n}^{2}}=\rho_{n}
$$

tend vers $3 \xi_{3}{ }^{2}\left(2 \xi_{1} \xi_{3}-\xi_{2}{ }^{2}\right)$. Posons $\mu=\xi_{3}{ }^{2}, \nu=\xi_{3}{ }^{3}, \rho=3 \xi_{3}{ }^{2}\left(2 \xi_{1} \xi_{3}-\xi_{2}{ }^{2}\right)$. Ce qui précède prouve que $(0, \mu, \nu, \rho) \in A_{1}$; posant de plus $\theta=-\xi_{2} / \xi_{3}$, on constate que

$$
\frac{1}{6} \frac{\rho}{\nu}+\frac{1}{2} \frac{\nu}{\mu} \theta^{2}=\xi_{1}, \quad-\frac{\nu}{\mu} \theta=\xi_{2}, \quad \frac{\nu}{\mu}=\xi_{3}
$$

donc $\left(\xi_{1}, \xi_{2}, \xi_{3}, \xi_{4}\right) \in E_{2}$.

(B) Supposons que

$$
\frac{1}{6} \frac{\rho_{n}}{\nu_{n}}+\frac{1}{2} \frac{\nu_{n}}{\mu_{n}} \theta_{n}^{2} \rightarrow \xi_{1}, \quad-\frac{\nu_{n}}{\mu_{n}} \theta_{n} \rightarrow \xi_{2}, \quad \frac{\nu_{n}}{\mu_{n}} \rightarrow \xi_{3}
$$

où $\left(0, \mu_{n}, \nu_{n}, \rho_{n}\right) \in A_{1}$, et où $\xi_{1}, \xi_{2}, \xi_{3}$ sont des nombres réels finis tels que $\xi_{3} \neq 0$. Comme $\nu_{n}{ }^{2}=\mu_{n}{ }^{3}$, on a $\nu_{n} / \mu_{n}=\nu_{n}^{\frac{1}{3}}$, donc $\mu_{n}$ et $\nu_{n}$ ont des limites finies $\mu=\xi_{3}{ }^{2}$ et $\nu=\xi_{3}{ }^{3}$. Alors, $\theta_{n}$ et $\rho_{n}$ ont des limites finies $\theta$ et $\rho$. On a $(0, \mu, \nu, \rho) \in A_{1}$, et

$$
\frac{1}{6} \frac{\rho}{\nu}+\frac{1}{2} \frac{\nu}{\mu} \theta^{2}=\xi_{1}, \quad-\frac{\nu}{\mu} \theta=\xi_{2}, \quad \frac{\nu}{\mu}=\xi_{3},
$$

donc $\left(\xi_{1}, \xi_{2}, \xi_{3}, 0\right) \in E_{2}$. Ceci achève la démonstration du lemme.

Revenons à la démonstration de la Proposition 6 . Soit $A_{1}$ une partie de $A$ fermée pour la topologie des paramètres. Soit $\left(\lambda_{0}, \mu_{0}, \nu_{0}, \rho_{0}\right)$ un point de $A$ n'appartenant pas à $A_{1}$. Soient $\rho_{1}, \theta$ deux nombres réels. Conservons les notations du Lemme 14 . Si $\lambda_{0} \neq 0$, le point

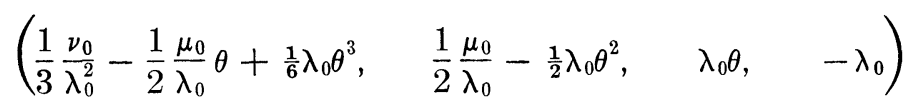

n'appartient pas à $E_{1} \cup E_{2}$, donc est extérieur à $E_{1} \cup E_{2}$. Si $\lambda_{0}=0$, le point

$$
\left(\frac{1}{6} \frac{\rho_{0}}{\nu_{0}}+\frac{1}{2} \frac{\nu_{0}}{\mu_{0}} \theta^{2}, \quad-\frac{\nu_{0}}{\mu_{0}} \theta, \quad \frac{\nu_{0}}{\mu_{0}}, 0\right)
$$


n'appartient pas à $E_{1} \cup E_{2}$, donc est extérieur à $E_{1} \cup E_{2}$. Dans les deux cas, il existe une $F \in \mathscr{P}\left(\Gamma_{5,5}\right)$ telle que $\hat{F}_{2345}$ soit nulle sur $\mathbf{R} \times\left(E_{1} \cup E_{2}\right)$ et non nulle au point

$$
\left(\begin{array}{ccccc}
\rho_{1}-\theta, & \frac{1}{3} \frac{\nu_{0}}{\lambda_{0}^{2}}-\frac{1}{2} \frac{\mu_{0}}{\lambda_{0}} \theta+\frac{1}{6} \lambda_{0} \theta^{3}, & \frac{1}{2} \frac{\mu_{0}}{\lambda_{0}}-\frac{1}{2} \lambda_{0} \theta^{2}, & \lambda_{0} \theta, & -\lambda_{0}
\end{array}\right)
$$

(resp. au point

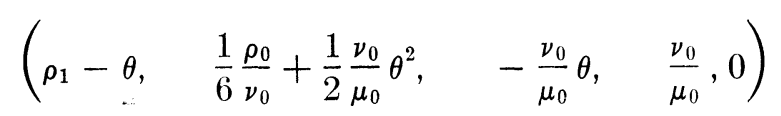

si $\left.\lambda_{0}=0\right)$. Alors,

$$
U_{\lambda_{0}, \mu_{0}, \nu_{0}, \rho_{0}}(F) \neq 0
$$

et $U_{\lambda, \mu, \nu, \rho}(F)=0$ pour $(\lambda, \mu, \nu, \rho) \in A_{1}$. Donc $U_{\lambda_{0}, \mu_{0}, \nu_{0}, \rho_{0}}$ est extérieur à $A_{1}$ pour la topologie canonique. Ceci prouve que $A_{1}$ est canoniquement fermé, et achève la démonstration de la proposition 6 .

Remarque. La mesure de Plancherel sur $A \subset \bar{\Gamma}_{5,5}$ est défine par la forme différentiell $w=\lambda^{-2} d \lambda d \mu d \nu$ (1, Proposition 9). Comme $2 \lambda^{-2} d \lambda d \mu d \nu=\nu^{-1} d \lambda d \mu d \rho$, $w$ est régulière sur tout $A$, contrairement à ce qui est dit dans (1), p. 322, 1. $18-21$.

8. Topologie de $\bar{\Gamma}_{5,6}$. D'après (1, Proposition 10), $\bar{\Gamma}_{5,6}$ est réunion de 4 sous-ensembles disjoints $A, B, C, D$ :

(1) $A$ est l'ensemble des $U_{\lambda}(\lambda \in \mathbf{R}, \lambda \neq 0)$; chaque $U_{\lambda}$ opère dans $L_{\mathbf{C}^{2}}{ }^{2}\left(\mathbf{R}^{2}\right)$ et est définie par la formule

$$
\begin{aligned}
& \left(U_{\lambda}(\gamma) f\right)\left(\theta_{1}, \theta_{2}\right) \\
& =\exp i \lambda\left(\rho_{5}-\rho_{4} \theta_{1}+\frac{1}{2} \rho_{2}^{2} \theta_{1}+\frac{1}{2} \rho_{3} \theta_{1}^{2}-\frac{1}{6} \rho_{2} \theta_{1}^{3}-\rho_{3} \theta_{2}+\rho_{2} \theta_{1} \theta_{2}\right) f\left(\theta_{1}+\rho_{1}, \theta_{2}+\rho_{2}\right) \\
& \quad\left(\gamma=\left(\rho_{1}, \ldots, \rho_{5}\right) \in \Gamma_{5,6}, \quad f \in L_{\mathbf{C}}^{2}\left(\mathbf{R}^{2}\right), \quad \theta_{1}, \theta_{2} \in \mathbf{R}\right) .
\end{aligned}
$$

(2) $B$ est l'ensemble des $V_{\lambda, \mu}(\lambda, \mu \in \mathbf{R}, \lambda \neq 0)$; chaque $V_{\lambda, \mu}$ opère dans $L_{\mathbf{C}}{ }^{2}(\mathbf{R})$ et est définie par la formule

$$
\left(V_{\lambda, \mu}(\gamma) f\right)(\theta)=\exp i\left(-\frac{1}{2} \frac{\mu}{\lambda} \rho_{2}+\lambda \rho_{4}-\lambda \rho_{3} \theta+\frac{1}{2} \lambda \rho_{2} \theta^{2}\right) f\left(\theta+\rho_{1}\right) .
$$

(3) $C$ est l'ensemble des $W_{\lambda}(\lambda \in \mathbf{R}, \lambda \neq 0)$; chaque $W_{\lambda}$ opère dans $L_{\mathbf{C}}{ }^{2}(\mathbf{R})$ et est définie par la formule

$$
\left(W_{\lambda}(\gamma) f\right)(\theta)=\exp i \lambda\left(\rho_{3}-\rho_{2} \theta\right) f\left(\theta+\rho_{1}\right) .
$$

(4) $D$ est l'ensemble des $X_{\lambda, \mu}(\lambda, \mu \in \mathbf{R})$; chaque $X_{\lambda, \mu}$ s'identifie à une fonction scalaire sur $\Gamma_{5,6}$ conformément à la relation

$$
X_{\lambda, \mu}(\gamma)=\exp i\left(\lambda \rho_{1}+\mu \rho_{2}\right) .
$$

D'après le Lemme $3, B \cup C \cup D$ est canoniquement fermé dans $\bar{\Gamma}_{5,6}$ et la topologie induite sur $B \cup C \cup D$ par la topologie canonique de $\bar{\Gamma}_{5,6}$ est la topologie canonique de $\bar{\Gamma}_{4}$. 
On définit sur $\bar{\Gamma}_{5,6}$ une topologie des paramètres.

Avec les notations habituelles, on a

(14) $\left(U_{\lambda}(F) f \mid g\right)$

$$
\begin{aligned}
& =\int \ldots \int F\left(\rho_{1}, \ldots, \rho_{5}\right) \exp i \lambda\left(\rho_{5}-\rho_{4} \theta_{1}+\frac{1}{2} \rho_{2}^{2} \theta_{1}+\frac{1}{2} \rho_{3} \theta_{1}^{2}-\frac{1}{6} \rho_{2} \theta_{1}^{3}\right. \\
& \left.-\rho_{3} \theta_{2}+\rho_{2} \theta_{1} \theta_{2}\right) f\left(\theta_{1}+\rho_{1}, \theta_{2}+\rho_{2}\right) \overline{g\left(\theta_{1}, \theta_{2}\right)} d \rho_{1} \ldots d \rho_{5} d \theta_{1} d \theta_{2} \\
& =\int \ldots \int F\left(\rho_{1}-\theta_{1}, \rho_{2}-\theta_{2}, \rho_{3}, \rho_{4}, \rho_{5}\right) \exp (i \lambda A) f\left(\rho_{1}, \rho_{2}\right) \overline{g\left(\theta_{1}, \theta_{2}\right)} d \rho_{1} \ldots d \rho_{5}
\end{aligned}
$$

$d \theta_{1} d \theta_{2}$

avec

$$
A=\rho_{5}-\rho_{4} \theta_{1}+\frac{1}{2}\left(\rho_{2}-\theta_{2}\right)^{2} \theta_{1}+\frac{1}{2} \rho_{3} \theta_{1}^{2}-\frac{1}{6}\left(\rho_{2}-\theta_{2}\right) \theta_{1}^{3}-\rho_{3} \theta_{2}+\left(\rho_{2}-\theta_{2}\right) \theta_{1} \theta_{2} .
$$

Donc $U_{\lambda}(F)$ est définie par le noyau

(15) $K_{\lambda}\left(\rho_{1}, \rho_{2}, \theta_{1}, \theta_{2}\right)$

$$
\begin{aligned}
& =\iiint F\left(\rho_{1}-\theta_{1}, \rho_{2}-\theta_{2}, \rho_{3}, \rho_{4}, \rho_{5}\right) \exp (i \lambda A) d \rho_{3} d \rho_{4} d \rho_{5} \\
& =\exp i \lambda\left(\frac{1}{2}\left(\rho_{2}-\theta_{2}\right)^{2} \theta_{1}-\frac{1}{6}\left(\rho_{2}-\theta_{2}\right) \theta_{1}^{3}+\left(\rho_{2}-\theta_{2}\right) \theta_{1} \theta_{2}\right) \\
& \qquad \hat{F}_{345}\left(\rho_{1}-\theta_{1}, \rho_{2}-\theta_{2}, \lambda \theta_{2}-\frac{1}{2} \lambda \theta_{1}^{2}, \lambda \theta_{1},-\lambda\right) .
\end{aligned}
$$

Lemme 15. Soit $F \in \mathscr{S}\left(\Gamma_{5,6}\right)$. La fonction $T \rightarrow\|T(F)\|$ est continue sur $\bar{\Gamma}_{5,6}$ pour la topologie des paramètres.

Démonstration analogue à celle du Lemme 12 .

Lemme 16. Soient $A_{1} \subset A, B_{1} \subset B, C_{1} \subset C, D_{1} \subset D$. On suppose $A_{1} \cup B_{1}$ $\cup C_{1} \cup D_{1}$ canoniquement fermé. Si 0 est adhérent à $A_{1}$ dans $\mathbf{R}$, on a $B_{1}=B$, $C_{1}=C, D_{1}=D$.

Démonstration. Soit $\beta_{1} \in \mathbf{R}-\{0\}$. Avec les notations habituelles, on a

$$
\begin{aligned}
& \left(U_{\lambda}(F) \mathscr{T}\left(-\frac{\beta_{1}}{\lambda},-\frac{\beta_{1}^{2}}{2 \lambda^{2}}\right) \mathscr{M}\left(\exp -i\left(\frac{1}{3} \frac{\beta_{1}^{3}}{\lambda^{2}} \theta_{2}+\frac{1}{2} \beta_{1} \theta_{2}^{2}\right)\right) f\right. \\
& \left.\mathscr{T}\left(-\frac{\beta_{1}}{\lambda},-\frac{\beta_{1}^{2}}{2 \lambda^{2}}\right) \mathscr{M}\left(\exp -i\left(\frac{1}{3} \frac{\beta_{1}^{3}}{\lambda^{2}} \theta_{2}+\frac{1}{2} \beta_{1} \theta_{2}^{2}\right)\right) g\right) \\
& =\int \ldots \int F\left(\rho_{1}, \ldots, \rho_{5}\right) \exp (i \Delta) \exp \left[-i\left(\frac{1}{3} \frac{\beta_{1}^{3}}{\lambda^{2}}\left(\theta_{2}-\frac{\beta_{1}^{2}}{2 \lambda^{2}}+\rho_{2}\right)\right.\right. \\
& \left.\left.+\frac{1}{2} \beta_{1}\left(\theta_{2}-\frac{\beta_{1}^{2}}{2 \lambda^{2}}+\rho_{2}\right)^{2}\right)\right] \\
& \exp \left[i\left(\frac{1}{3} \frac{\beta_{1}^{3}}{\lambda^{2}}\left(\theta_{2}-\frac{\beta_{1}^{2}}{2 \lambda^{2}}\right)+\frac{1}{2} \beta_{1}\left(\theta_{2}-\frac{\beta_{1}^{2}}{2 \lambda^{2}}\right)^{2}\right] f\left(\theta_{1}-\frac{\beta_{1}}{\lambda}+\rho_{1}, \theta_{2}-\frac{\beta_{1}^{2}}{2 \lambda^{2}}+\rho_{2}\right)\right. \\
& \overline{g\left(\theta_{1}-\frac{\beta_{1}}{\lambda}, \theta_{2}-\frac{\beta_{1}^{2}}{2 \lambda^{2}}\right)} d \rho_{1} \ldots d \rho_{5} d \theta_{1} d \theta_{2}
\end{aligned}
$$




$$
\begin{array}{r}
=\int \ldots \int F\left(\rho_{1}, \ldots, \rho_{5}\right) \exp (i \Delta) \exp \left[-i\left(\frac{1}{3} \frac{\beta_{1}^{3}}{\lambda^{2}} \rho_{2}+\beta_{1}\left(\theta_{2}-\frac{\beta_{1}^{2}}{2 \lambda^{2}}\right) \rho_{2}\right.\right. \\
\left.\left.+\frac{1}{2} \beta_{1} \rho_{2}^{2}\right)\right] f\left(\theta_{1}-\frac{\beta_{1}}{\lambda}+\rho_{1}, \theta_{2}-\frac{\beta_{1}^{2}}{2 \lambda^{2}}+\rho_{2}\right) \overline{g\left(\theta_{1}-\frac{\beta_{1}}{\lambda}, \theta_{2}-\frac{\beta_{1}^{2}}{2 \lambda^{2}}\right)} \\
d \rho_{1} \ldots d \rho_{5} d \theta_{1} d \theta_{2} \\
=\int \ldots \int F\left(\rho_{1}, \ldots, \rho_{5}\right) \exp \left(i \Delta^{\prime}\right) f\left(\theta_{1}+\rho_{1}, \theta_{2}+\rho_{2}\right) \overline{g\left(\theta_{1}, \theta_{2}\right)} d \rho_{1} \ldots d \rho_{5} d \theta_{1} d \theta_{2}
\end{array}
$$

avec

$$
\begin{aligned}
\Delta^{\prime}= & \lambda \rho_{5}-\lambda \rho_{4}\left(\theta_{1}+\frac{\beta_{1}}{\lambda}\right)+\frac{1}{2} \lambda \rho_{2}^{2}\left(\theta_{1}+\frac{\beta_{1}}{\lambda}\right)+\frac{1}{2} \lambda \rho_{3}\left(\theta_{1}+\frac{\beta_{1}}{\lambda}\right)^{2}-\frac{1}{6} \lambda \rho_{2}\left(\theta_{1}+\frac{\beta_{1}}{\lambda}\right)^{3} \\
& \quad-\lambda \rho_{3}\left(\theta_{2}+\frac{\beta_{1}^{2}}{2 \lambda^{2}}\right)+\lambda \rho_{2}\left(\theta_{1}+\frac{\beta_{1}}{\lambda}\right)\left(\theta_{2}+\frac{\beta_{1}^{2}}{2 \lambda^{2}}\right)-\frac{1}{3} \frac{\beta_{1}^{3}}{\lambda^{2}} \rho_{2}-\beta_{1} \rho_{2} \theta_{2}-\frac{1}{2} \beta_{1} \rho_{2}^{2} \\
= & \lambda\left(\rho_{5}-\rho_{4} \theta_{1}+\frac{1}{2} \rho_{2}^{2} \theta_{1}+\frac{1}{2} \rho_{3} \theta_{1}^{2}-\frac{1}{6} \rho_{2} \theta_{1}^{3}-\rho_{3} \theta_{2}+\rho_{2} \theta_{1} \theta_{2}\right)-\beta_{1} \rho_{4}+\beta_{1} \rho_{3} \theta_{1}-\frac{1}{2} \beta_{1} \rho_{2} \theta_{1}^{2} .
\end{aligned}
$$

Donc, quand $\lambda \rightarrow 0$, l'intégrale étudiée a pour limite

$$
\begin{aligned}
& \int \ldots \int F\left(\rho_{1}, \ldots, \rho_{5}\right) \exp i\left(-\beta_{1} \rho_{4}+\beta_{1} \rho_{3} \theta_{1}-\frac{1}{2} \beta_{1} \rho_{2} \theta_{1}^{2}\right) f\left(\theta_{1}+\rho_{1}, \theta_{2}+\rho_{2}\right) \\
& \overline{g\left(\theta_{1}, \theta_{2}\right)} d \rho_{1} \ldots d \rho_{5} d \theta_{1} d \theta_{2} .
\end{aligned}
$$

Identifions, comme au paragraphe 6, l'espace $L_{\mathbf{C}}{ }^{2}\left(\mathbf{R}^{2}\right)$ à l'intégrale hilbertienne

$$
\int^{\oplus} \mathfrak{S}_{\theta_{2}} d \theta_{2}
$$

où

$$
\mathfrak{S}_{\theta_{2}}=L_{\mathbf{C}}^{2}(\mathbf{R})
$$

pour tout $\theta_{2} \in \mathbf{R}$. Le champ d'opérateurs

$$
\theta_{2} \rightarrow V_{-\beta_{1},-2 \beta_{1} \theta_{2}}(F)
$$

est fortement continu (et même beaucoup mieux!) et il définit dans $L_{\mathbf{C}^{2}}{ }^{2}\left(\mathbf{R}^{2}\right)$ un opérateur $S$ tel que

$$
\begin{aligned}
(S f \mid g) & =\int\left(V_{-\beta_{1},-2 \beta_{1} \theta_{2}}(F) f_{\theta_{2}} \mid g_{\theta_{2}}\right) d \theta_{2} \\
& =\int \ldots \int F\left(\rho_{1}, \ldots, \rho_{5}\right) \exp i\left(-\rho_{2} \theta_{2}-\beta_{1} \rho_{4}+\beta_{1} \rho_{3} \theta_{1}-\frac{1}{2} \beta_{1} \rho_{2} \theta_{1}^{2}\right) \\
& f\left(\theta_{1}+\rho_{1}, \theta_{2}\right) \overline{g\left(\theta_{1}, \theta_{2}\right)} d \rho_{1} \ldots d \rho_{5} d \theta_{1} d \theta_{2} .
\end{aligned}
$$

Désignant encore par $\mathscr{F}_{2}$ la transformation de Fourier par rapport à la 2e variable dans $L_{\mathbf{C}^{2}}{ }^{2}\left(\mathbf{R}^{2}\right)$, on obtient 


$$
\begin{array}{r}
\left(\mathscr{F}_{2} S \mathscr{F}_{2}^{-1} f \mid g\right)=\int \ldots \int F\left(\rho_{1}, \ldots, \rho_{5}\right) \exp i\left(-\beta_{1} \rho_{4}+\beta_{1} \rho_{3} \theta_{1}-\frac{1}{2} \beta_{1} \rho_{2} \theta_{1}^{2}\right) \\
f\left(\theta_{1}+\rho_{1}, \theta_{2}+\rho_{2}\right) \overline{g\left(\theta_{1}, \theta_{2}\right)} d \rho_{1} \ldots d \rho_{5} d \theta_{1} d \theta_{2} .
\end{array}
$$

En définitive, quand $\lambda \rightarrow 0$, l'opérateur

$$
\begin{aligned}
\mathscr{M}\left(\exp i\left(\frac{1}{3} \frac{\beta_{1}^{3}}{\lambda^{2}} \theta_{2}+\frac{1}{2} \beta_{1} \theta_{2}^{2}\right)\right) \mathscr{T}\left(\frac{\beta_{1}}{\lambda}, \frac{\beta_{1}^{2}}{2 \lambda^{2}}\right) U_{\lambda}(F) \mathscr{T}\left(\frac{\beta_{1}}{\lambda}, \frac{\beta_{1}^{2}}{2 \lambda^{2}}\right)^{-1} \\
\mathscr{M}\left(\exp i\left(\frac{1}{3} \frac{\beta_{1}^{3}}{\lambda^{2}} \theta_{2}+\frac{1}{2} \beta_{1} \theta_{2}^{2}\right)\right)^{-1}
\end{aligned}
$$

tend faiblement vers $\mathscr{F}_{2} S_{2} \mathscr{F}^{-1}$. Par suite

$$
\lim _{\lambda \rightarrow 0}\left\|U_{\lambda}(F)\right\| \geqslant \sup _{\theta_{2} \in \mathbf{R}}\left\|V_{-\beta_{1},-2 \beta_{1} \theta_{2}}(F)\right\|=\sup _{\mu \in \mathbf{R}}\left\|V_{-\beta_{1}, \mu}(F)\right\| .
$$

Donc $V_{-\beta_{1}, \mu} \in B_{1}$. Comme $\beta_{1}$ et $\mu$ sont arbitraires $\left(\beta_{1} \neq 0\right)$, on a $B_{1}=B$. D'après la Proposition $2, B$ est partout dense dans $B \cup C \cup D$ pour la topologie canonique, donc $C_{1}=C, D_{1}=D$.

Proposition 7. Les ensembles canoniquement fermés de $\bar{\Gamma}_{5,6}$ sont les ensembles $A_{1} \cup B_{1} \cup C_{1} \cup D_{1}\left(A_{1} \subset A, B_{1} \subset B, C_{1} \subset C, D_{1} \subset D\right)$ possédant les propriétés suivantes:

(1) $A_{1}$ est fermé dans $A$ pour la topologie des paramètres;

(2) $B_{1} \cup C_{1} \cup D_{1}$ est canoniquement fermé dans $B \cup C \cup D=\bar{\Gamma}_{4}$;

(3) Si 0 est adhérent à $A_{1}$ dans $\mathbf{R}$, on a $B_{1}=B, C_{1}=C, D_{1}=D$.

Démonstration. Supposons remplies les conditions (1), (2), et (3) de la proposition. Soit $T \in \bar{\Gamma}_{5,6}$ avec $T \notin A_{1} \cup B_{1} \cup C_{1} \cup D_{1}$. Il s'agit de construire une $F \in \mathscr{S}\left(\Gamma_{5,6}\right)$ telle que $T(F) \neq 0$ et $T^{\prime}(F)=0$ pour $T^{\prime} \in A_{1}$.

(A) Supposons $T=U_{\lambda_{0}} \notin A_{1}$. Il existe $F \in \mathscr{S}\left(\Gamma_{5,6}\right)$ telle que $\hat{F}_{345}$ soit nulle lorsque la 5 e variable appartient à $A_{1}$ et non nulle lorsque la 5 e variable a pour valeur $\lambda_{0}$. Alors $U_{\lambda 0}(F) \neq 0$ et $U_{\lambda}(F)=0$ pour $\lambda \in A_{1}$.

(B) Supposons $T=V_{\lambda_{0}, \mu_{0}} \notin B_{1}$. Alors, d'après la condition (3) de la proposition, 0 est non adhérent à $A_{1}$ dans $\mathbf{R}$. Donc il existe $F \in \mathscr{S}\left(\bar{\Gamma}_{5,6}\right)$ telle que $\hat{F}_{2345}$, et par suite $\hat{F}_{345}$, soient nulles lorsque la 5e variable appartient à $A_{1}$, et telle que la fonction

$$
\left(\rho_{1}, \theta\right) \rightarrow \hat{F}_{2345}\left(\rho_{1}-\theta, \frac{1}{2} \frac{\mu_{0}}{\lambda_{0}}-\frac{1}{2} \lambda_{0} \theta^{2}, \lambda_{0} \theta,-\lambda_{0}, 0\right)
$$

ne soit pas identiquement nulle. Alors $V_{\lambda_{0}, \mu_{0}}(F) \neq 0$, et $U_{\lambda}(F)=0$ pour $\lambda \in A_{1}$.

(C) On raisonne de manière analogue pour $T=W_{\lambda_{1}} \notin C_{1}$ ou $T=X_{\lambda_{0}, \mu_{0}} \notin D_{1}$.

\section{Etude des caractères.}

Proposition 8. Soit $\Gamma_{4}{ }^{\prime}$ le sous-groupe de $\Gamma_{4}$ d'équation $\rho_{1}=0$. Le caractère de $U_{\lambda, \mu}$ est une mesure concentrée sur $\Gamma_{4}{ }^{\prime}$. Sa restriction $\grave{a} \Gamma_{4}{ }^{\prime}$ est définie par la densité 


$$
(1 \pm i)\left(\frac{\pi}{\left|\lambda \rho_{2}\right|}\right)^{\frac{1}{2}} \exp \frac{1}{2} i\left(\lambda \frac{2 \rho_{2} \rho_{4}-\rho_{3}^{2}}{\rho_{2}}-\frac{\mu}{\lambda} \rho_{2}\right)
$$

où il faut prendre le signe + pour $\lambda \rho_{2}>0$, et le signe - pour $\lambda \rho_{2}<0$.

Démonstration. On a, pour $F \in \mathscr{S}\left(\Gamma_{4}\right)$,

$$
\operatorname{tr}\left(U_{\lambda, \mu}(F)\right)=\int \hat{F}_{234}\left(0, \frac{1}{2} \frac{\mu}{\lambda}-\frac{1}{2} \lambda \theta^{2}, \lambda \theta,-\lambda\right) d \theta .
$$

Donc le caractère de $U_{\lambda, \mu}$ est l'extension à $\Gamma_{4}$ d'une distribution définie sur $\Gamma_{4}{ }^{\prime}$. Identifiant $\Gamma_{4}{ }^{\prime}$ à $\mathbf{R}^{3}$ grâce au système de coordonnées $\left(\rho_{2}, \rho_{3}, \rho_{4}\right)$ on voit que cette distribution est la transformée de Fourier $\mathscr{F} m$ de la mesure $m$ à croissance lente définie par la formule

$$
m(f)=\int f\left(\frac{1}{2} \frac{\mu}{\lambda}-\frac{1}{2} \lambda \theta^{2}, \lambda \theta,-\lambda\right) d \theta
$$

$\left(f\right.$, fonction continue à support compact dans $\left.\mathbf{R}^{3}\right)$. Soit $m_{T}$ la mesure à support compact définie par la formule

$$
m_{T}(f)=\int_{-T}^{T} f\left(\frac{1}{2} \frac{\mu}{\lambda}-\frac{1}{2} \lambda \theta^{2}, \lambda \theta,-\lambda\right) d \theta .
$$

Quand $T \rightarrow+\infty, m_{T}$ tend vers $m$ au sens des distributions tempérées. Donc $\mathscr{F} m_{T}$ tend vers $\mathscr{F} m$ au sens des distributions tempérées. Or, $\mathscr{F} m_{T}$ est la fonction

$$
\left(\rho_{2}, \rho_{3}, \rho_{4}\right) \rightarrow \int_{-T}^{+T} \exp -i\left[\left(\frac{1}{2} \frac{\mu}{\lambda}-\frac{1}{2} \lambda \theta^{2}\right) \rho_{2}+\lambda \theta \rho_{3}-\lambda \rho_{4}\right] d \theta .
$$

Nous allons vérifier que, quand $T \rightarrow+\infty$, cette fonction converge simplement vers la fonction (16), en restant majorée en module par une fonction localement intégrable fixe. On en conclura que la fonction (17) tend vers la fonction (16) au sens des distributions, donc que la fonction (16) est la distribution $\mathscr{F} \mathrm{m}$ cherchée.

Supposons $\lambda \rho_{2}>0$. Dans l'intégrale (17), faisons le changement de variables $\left(\frac{1}{2} \lambda \rho_{2}\right)^{\frac{1}{2}}\left(\theta-\rho_{3} / \rho_{2}\right)=\zeta$. Elle devient

$$
\begin{aligned}
\int_{\left(\frac{\lambda \rho_{2}}{2}\right)^{\frac{1}{2}}\left(-T-\rho_{3} / \rho_{2}\right)}^{\left(\frac{\lambda \rho_{2}}{)^{\frac{1}{2}}\left(T-\rho_{3} / \rho_{2}\right)}\right.} \exp i\left[\zeta^{2}-\frac{1}{2} \lambda \frac{\rho_{3}^{2}}{\rho_{2}}-\frac{1}{2} \frac{\mu}{\lambda} \rho_{2}+\lambda \rho_{4}\right]\left(\frac{2}{\lambda \rho_{2}}\right)^{\frac{1}{2}} d \zeta \\
=\left(\frac{2}{\lambda \rho_{2}}\right)^{\frac{1}{2}} \exp \frac{i}{2}\left(\lambda \frac{2 \rho_{2} \rho_{4}-\rho_{3}^{2}}{\rho_{2}}-\frac{\mu}{\lambda} \rho_{2}\right) \int_{\left(\frac{\lambda \rho_{2}}{2}\right)^{\frac{1}{2}}\left(-T-\rho_{3} / \rho_{2}\right)}^{\left(\frac{\lambda \rho_{2}}{2}\right)^{\frac{1}{2}}\left(T-\rho_{3} / \rho_{2}\right)} \exp \left(i \zeta^{2}\right) d \zeta .
\end{aligned}
$$

Or, un calcul élémentaire montre que

$$
\left|\int_{a}^{b} \exp \left(i \zeta^{2}\right) d \zeta\right| \leqslant 4
$$

quels que soient $a$ et $b$. Et, quand $T \rightarrow+\infty$, l'intégrale tend vers

$$
\int_{-\infty}^{+\infty} \exp \left(i \zeta^{2}\right) d \zeta=\sqrt{\frac{\pi}{2}}(1+i) .
$$


La proposition est donc vérifiée pour $\lambda \rho_{2}>0$. On procède de façon analogue pour $\lambda \rho_{2}<0$.

Proposition 9. Soit $\Gamma_{5,5}$ le sous-groupe de $\Gamma_{5,5}$ d'équation $\rho_{1}=0$. Pour $\lambda \neq 0$, le caractère de $U_{\lambda, \mu, \nu, \rho}$ est une mesure concentrée sur $\Gamma_{5,5}{ }^{\prime}$. Sa restriction $\grave{a} \Gamma_{5,5}{ }^{\prime}$ est définie par la densité d suivante; on pose

$$
\begin{aligned}
& \alpha=\frac{1}{2}\left(\frac{6}{\lambda \rho_{2}}\right)^{\frac{1}{3}}\left(\lambda \frac{2 \rho_{2} \rho_{4}-\rho_{3}^{2}}{\rho_{2}}-\frac{\mu}{\lambda} \rho_{2}\right) \\
& d= \pm\left(\frac{6}{\lambda \rho_{2}}\right)^{\frac{1}{3}} \exp \frac{i}{3}\left(\frac{3 \rho_{2}^{2} \rho_{5}-3 \rho_{2} \rho_{3} \rho_{4}+\rho_{3}^{3}}{\rho_{2}^{2}}-\frac{\nu}{\lambda^{2}} \rho_{2}\right) \Delta\left(\rho_{2}, \rho_{3}, \rho_{4}\right)
\end{aligned}
$$

où il faut prendre le signe + pour $\lambda \rho_{2}>0$, le signe - pour $\lambda \rho_{2}<0$, et où

$$
\begin{aligned}
& \Delta=\frac{2}{3} \alpha^{\frac{1}{2}} K_{1 / 3}\left(\frac{2 \alpha^{3 / 2}}{3 \sqrt{ } 3}\right) \quad \text { pour } \quad \alpha>0 \\
& \Delta=\frac{2 \pi}{3}\left(\frac{1}{3} \alpha\right)^{\frac{1}{2}}\left[J_{1 / 3}\left(\frac{2}{3} \alpha\left(\frac{1}{3} \alpha\right)^{\frac{1}{2}}\right)+J_{-1 / 3}\left(\frac{2}{3} \alpha\left(\frac{1}{3} \alpha\right)^{\frac{1}{2}}\right)\right] \quad \text { pour } \quad \alpha<0 .
\end{aligned}
$$

$\left(J_{1 / 3}, J_{-1 / 3}, K_{1 / 3}\right.$ sont les notations classiques pour les fonctions de Bessel.)

Démonstration. On a, pour $F \in \mathscr{S}\left(\Gamma_{5,5}\right)$ et $\lambda \neq 0$,

$$
\operatorname{tr}\left(U_{\lambda, \mu, \nu, \rho}(F)\right)=\int \hat{F}_{2345}\left(0, \frac{1}{3} \frac{\nu}{\lambda^{2}}-\frac{1}{2} \frac{\mu}{\lambda} \theta+\frac{1}{6} \lambda \theta^{3}, \frac{1}{2} \frac{\mu}{\lambda}-\frac{1}{2} \lambda \theta^{2}, \lambda \theta,-\lambda\right) d \theta .
$$

Donc le caractère de $U_{\lambda, \mu, \nu, \rho}$ et l'extension à $\Gamma_{5,5}$ d'une distribution définie sur $\Gamma_{5,5}{ }^{\prime}$. Identifiant $\Gamma_{5,5}{ }^{\prime}$ à $\mathbf{R}^{4}$ grâce au système de coordonnées $\left(\rho_{2}, \rho_{3}, \rho_{4}, \rho_{5}\right)$, on voit que cette distribution est la transformée de Fourier $\mathscr{F} m$ de la mesure $m$ à croissance lente définie par la formule

$$
m(f)=\int f\left(\frac{1}{3} \frac{\nu}{\lambda^{2}}-\frac{1}{2} \frac{\mu}{\lambda} \theta+\frac{1}{6} \lambda \theta^{3}, \frac{1}{2} \frac{\mu}{\lambda}-\frac{1}{2} \lambda \theta^{2}, \lambda \theta,-\lambda\right) d \theta
$$

$\left(f\right.$, fonction continue à support compact dans $\left.\mathbf{R}^{4}\right)$. Procédant comme pour la Proposition 8, on est amené à considérer l'intégrale

$$
\int_{-T}^{+T} \exp -i\left[\left(\frac{1}{3} \frac{\nu}{\lambda^{2}}-\frac{1}{2} \frac{\mu}{\lambda} \theta+\frac{1}{6} \lambda \theta^{3}\right) \rho_{2}+\left(\frac{1}{2} \frac{\mu}{\lambda}-\frac{1}{2} \lambda \theta^{2}\right) \rho_{3}+\lambda \theta \rho_{4}-\lambda \rho_{5}\right] d \theta .
$$

Supposons $\rho_{2} \neq 0$. Faisant, dans cette intégrale, le changement de variable

$$
\left(\frac{1}{6} \lambda \rho_{2}\right)^{\frac{1}{3}}\left(\theta-\frac{\rho_{3}}{\rho_{2}}\right)=\zeta
$$

elle devient

$$
\begin{aligned}
\left(\frac{6}{\lambda \rho_{2}}\right)^{\frac{1}{3}} \exp \frac{i}{3}\left(\lambda \frac{3 \rho_{2}^{2} \rho_{5}-3 \rho_{2} \rho_{3} \rho_{4}+\rho_{3}^{3}}{\rho_{2}^{2}}-\frac{\nu}{\lambda^{2}} \rho_{2}\right) \int_{u}^{v} \exp -i\left[\zeta^{3}+\frac{1}{2}\left(\frac{6}{\lambda \rho_{2}}\right)^{\frac{1}{3}}\right. & \left.\left(\lambda \frac{2 \rho_{2} \rho_{4}-\rho_{3}^{2}}{\rho_{2}}-\frac{\mu}{\lambda} \rho_{2}\right) \zeta\right] d \zeta
\end{aligned}
$$


avec

$$
u=\left(\frac{1}{6} \lambda \rho_{2}\right)^{\frac{1}{3}}\left(-T-\frac{\rho_{3}}{\rho_{2}}\right) \quad v=\left(\frac{1}{6} \lambda \rho_{2}\right)^{\frac{1}{3}}\left(T-\frac{\rho_{3}}{\rho_{2}}\right) .
$$

Une majoration élémentaire montre que

$$
\left|\int_{a}^{b} \exp -i\left(\zeta^{3}+\alpha \zeta\right) d \zeta\right| \leqslant 10
$$

quels que soient $a, b, \alpha$. Donc $\mathscr{F} m$ est la fonction

$$
\pm\left(\frac{6}{\lambda \rho_{2}}\right)^{\frac{1}{3}} \exp \frac{i}{3}\left(\lambda \frac{3 \rho_{2}^{2} \rho_{5}-3 \rho_{2} \rho_{3} \rho_{4}+\rho_{3}^{3}}{\rho_{2}^{2}}-\frac{\nu}{\lambda^{2}} \rho_{2}\right) \int_{-\infty}^{+\infty} \exp -i\left(\zeta^{3}+\alpha \zeta\right) d \zeta
$$

où il faut prendre le signe + pour $\lambda \rho_{2}>0$, le signe - pour $\lambda \rho_{2}<0$. Or, on a (intégrale d'Airy)

$$
\int_{-\infty}^{+\infty} \exp i\left(\zeta^{3}+\alpha \zeta\right) d \zeta=\left\{\begin{array}{l}
\frac{2}{3} \alpha^{\frac{1}{2}} K_{1 / 3}\left(2\left(\frac{1}{3} \alpha\right)^{3 / 2}\right)(\alpha>0) \\
2 \pi / 3\left(\frac{1}{3} \alpha\right)^{\frac{1}{2}}\left[J_{1 / 3}\left(2\left(\frac{1}{3} \alpha\right)^{3 / 2}\right)+J_{-1 / 3}\left(2\left(\frac{1}{3} \alpha\right)^{3 / 2}\right)\right](\alpha<0) .
\end{array}\right.
$$

D'où la proposition.

Je dois à Delsarte la remarque que la limite de l'intégrale (18), quand $T \rightarrow+\infty$, peut se calculer explicitement.

\section{BiBLIOGRAPHIE}

1. J. Dixmier, Sur les représentations unitaires des groupes de Lie nilpotents. III, Can. J. Math., 10 (1958), 321-348.

2. - Sur les représentations unitaires des groupes de Lie nilpotents. V, Bull. Soc. Math. France, 87 (1959), 65-79.

3. J. M. G. Fell, The dual spaces of $C^{*}$-algebras, à paraître aux Trans. Amer. Math. Soc.

4. R. Godement, Les fonctions de type positif et la théorie des groupes, Trans. Amer. Math. Soc., 63 (1948), 1-84.

\section{Institut Henri Poincaré}

